\title{
Simulation of primary atomization with an octree adaptive mesh refinement and VOF method
}

\author{
Daniel Fuster ${ }^{\mathrm{a}, \mathrm{b}}$, Anne Bagué $\mathrm{e}^{\mathrm{a}, \mathrm{b}}$, Thomas Boeck ${ }^{\mathrm{c}}$, Luis Le Moyne ${ }^{\mathrm{a}, \mathrm{b}}$, Anthony \\ Leboissetier $^{\mathrm{d}}$, Stéphane Popinet ${ }^{\mathrm{e}}$, Pascal Ray ${ }^{\mathrm{a}, \mathrm{b}}$, Ruben Scardovellif ${ }^{\mathrm{f}}$, Stéphane \\ Zaleski ${ }^{a, b}$ \\ ${ }^{a}$ UPMC Univ Paris 06, UMR 7190, Institut Jean Le Rond d'Alembert, F-75005 Paris, \\ France. \\ ${ }^{b}$ CNRS, UMR 7190, Institut Jean Le Rond d'Alembert, F-75005 Paris, France. \\ ${ }^{c}$ Fachgebiet Thermo- und Fluiddynamik, TU Ilmenau, P.O. Box 100565, 98684 Ilmenau, \\ Germany \\ ${ }^{d}$ NASA GISS, 2880 Broadway, New York, NY 10025, USA. \\ ${ }^{e}$ NIWA, P.O. Box 14-901, Kilbirnie, Wellington, New Zealand. \\ ${ }^{f}$ DIENCA - Lab. di Montecuccolino, University of Bologna, Bologna, Italy.
}

\begin{abstract}
We present different simulations of primary atomization using an adaptive Volumeof-Fluid method based on octree meshes. The use of accurate numerical schemes for mesh adaptation, Volume-of-Fluid advection and balanced force surface tension calculation implemented in Gerris, the code used to perform the simulations included in this work, has made possible to carry out accurate simulations with characteristic scales spreading over several orders of magnitude. The code is validated by comparisons with the temporal linear theory for moderate density and viscosity ratios, which basically corresponds to atomization processes in high pressure chambers. In order to show the potential of the code in different scenarios related to atomization, preliminary results are shown in relation with the study of the two-dimensional and 3D temporal and spatial problem, the influence of the injector and the vortex generated inside the chamber, and the effect of swirling at high Reynolds numbers.
\end{abstract}

Key words: atomization, numerical simulation, Gerris, VOF, two-phase mixing layer, Kelvin-Helmholtz

PACS: $47.11 .+\mathrm{j}$

\section{Introduction}

The breakup of liquid masses by high-speed air streams is an amazingly complex phenomenon that occurs in many natural and man-made circumstances. For instance, spume formation at the top of sea-wave crests corresponds to the generation of a complex mixture of droplet spray, bubbles and wavelets induced by the relative air velocity. In the industrial domain, liquid-fuel combustion 
in many types of engines or furnaces requires the atomization of the fluid before evaporation and combustion can occur. This is of particular interest to the automotive and aerospace industry, as the quality of the combustion and thus pollutant generation depend on the characteristics of fuel atomization. In certain medical devices, drug delivery is assisted by spray formation. Many other domains are of interest: atomization is also used for painting, is relevant to "churn" liquid-gas flows in pipes and for powder production out of liquid metals.

We focus in this paper on injector technology, and particularly on devices where atomization results from the interplay of classical hydrodynamic phenomena, excluding interesting but more complex effects such as cavitation, electrodynamic forces or compressibility. Several mechanisms may be active in generating atomization in these injector systems. In certain types of injectors, such as swirling injectors, thin films are created that get thinner as they flow downstream. The stretching of the film may also be enhanced by various instabilities, but will in any case reach a level where the film breaks and ligaments are formed. In injectors without swirl two regimes may be observed at high velocity: close to the injector exit, relatively small ligaments or droplets are seen to detach from the jet. Further downstream, large scale instabilities of the jet may be observed, leading to the formation of large droplets.

The mechanisms leading to ligament and droplet formation are still the object of active research. At least two competing mechanisms are on offer. The first mechanism amounts to a scenario proposed by Faeth et al. (1995) in which a sufficient level of turbulence in the liquid phase upstream of the nozzle will deform the interface and lead to breakup. More precisely, if a turbulent eddy has sufficient energy it may defeat the stabilizing effect of surface tension and create a ligament. The second mechanism involves the gas phase in an essential way. The flow in a small region of the jet's surface is approximated by a twophase mixing layer. The stability of the corresponding two-phase parallel base flow is studied, leading to instabilities when the relative velocity of gas and liquid is sufficiently high compared to surface tension. At the time of writing, the general understanding of the phenomenon is not sufficiently advanced to decide what is the interplay between the influence of turbulence generated upstream and mixing layer instability.

As for many flow stability problems, the analysis is complex, and one needs to distinguish between linear and nonlinear effects, convective and absolute instability, normal-mode and transient growth, and delicate effects of viscosity and three-dimensionality even at high Reynolds numbers. The absolute/convective debate is particularly important: if the instability is convective, the system is a noise amplifier and the characteristics of background turbulence are essential.

The final droplet size has been the subject of much speculation. In many theories, only the initial development of a two-dimensional instability is pre- 
dicted. It then remains to explain how the resulting two dimensional sheets evolve into three-dimensional structures that lead to fingers, tubular ligaments and eventually droplets. A famous scenario assumes that the tip of the sheet develops a Taylor-Culick rim, which then detaches and breaks due to Plateau's and Rayleigh's jet instability (Dombrowski and Johns, 1963). Many other mechanisms have been proposed, some of them involving a secondary instability of the sheet.

Experimental studies can succeed in part to verify the various theories but only up to a certain point. For atomization with co-flowing gas and liquid streams, the predictions of inviscid linear theory yield the experimentally-observed exponent for the frequency dependence on velocity (Ben Ravana, 2007) but miss the exact value of the frequency by a factor of three to ten. The origins of this discrepancy are hard to analyze. One possible explanation would be the influence of preexisting modes upstream of the injector that would provide an initial perturbation of well-defined frequency.

To further our understanding of these phenomena numerical simulation offers several advantages. The simulation approximates the solution of the NavierStokes equations in a set-up that is an idealized model of the real experiments. How idealized the model is the choice of the investigator. For instance one may wish to study only two-dimensional flow, or flow without any turbulence upstream of the injection, or flow with well-defined mean velocity profiles at the inflow boundary condition. Numerical simulation then offers the possibility to serve as an intermediate step between theory and experiment, allowing to verify or falsify assumptions about the underlying mechanisms of atomization.

Early numerical studies of atomization started from a drastic simplifications of the problem. Rangel and Sirignano (1988) have studied the dynamics of vortex sheets in inviscid flow, showing the growth of the Kelvin-Helmholtz instability into the non-linear regime. Keller et al. (1994) (also reported by Scardovelli and Zaleski (1999)) using the Volume-of-Fluid method and Tauber et al. (2002) and Tauber and Trvggvason (2000) using front tracking, performed twodimensional simulations of the Navier-Stokes equations, also starting from a discontinuous velocity field and looking at the evolution in time of the instability in a spatially periodic domain, a set-up similar to that used in the temporal instability theory. Similar "temporal" two-dimensional simulations were reported recently by Boeck and Zaleski (2005) and Boeck et al. (2007) showing agreement over one to two decades of amplitude with the viscous linear stability theory and investigating the effect of boundary layers in the base flow configuration. Three-dimensional temporal simulations were reported by Zaleski et al. (1997) using the Volume-of-Fluid method and by Tauber (2002) using FrontTracking. These studies have shown the formation of fingers on top of unstable sheets but have not systematically studied the characteristics of ligament formation because of the excessive demands on computer time. Much more realistic spatial simulations are those where the fluid enters the simulation at 
one boundary mimicking the injector exit and leaves at the other boundaries after the instabilities have spatially developed. Such simulations were reported recently by Bianchi et al. (2005); De Villiers et al. (2004); Ménard et al. (2007); Gorokhovski and Herrmann (2008).

Although detailed 3D simulations are becoming possible, they are still limited by severe numerical challenges. To understand why it is useful to review the progress of numerical simulation methods for flows with liquid gas interfaces. Three main methods and a number of variants compete. Front-Tracking approximates the position of the interface using marker particles and interpolates between them in various ways, most typically using a triangular mesh in 3D simulations. This technique is simple in principle but has some disadvantages: the remeshing of the surface is necessary as the interface gets deformed, and the intersection of the surface with the grid and with itself is complex to manage. Finally, jet or droplet breakup requires a criterion for remeshing with a different topology at appropriately selected times thin tubular region or sheet-like regions. Determining the appropriate time and location of the break is one of the major difficulties. By contrast the two other methods handle change of topology automatically when the sheets or tube become as thin as the grid spacing. The Level-Set method advects a smooth function whose zero level set corresponds to the interface. Resetting the level set at regular intervals is necessary and is done by setting the advected function to be the signed distance function to the interface. The Volume-of-Fluid method tracks the volume fraction of liquid in each cell. Volume-of-Fluid methods are in some steps of the algorithm more complex to program but are typically be more accurate than level sets. Hybrid methods have also been developed such as marker-level-set, marker-VOF or level-set VOF. The main motivation for coupling with level-set methods seems to us to be the possibility of introducing a smoother function that yields the second order derivatives needed for the computation of surface tension. However using height functions as advocated by Cummins et al. (2005); Francois et al. (2006) and by one of us (Popinet, 2007) yields even more accurate results. Most methods run into difficulties when the density ratios and the relative strength of surface tension compared to other forces becomes large, which is the case for air-water systems, which are hence most of the time treated in the free-surface approximation. For atomizing systems, however, the role of aerodynamic friction is essential and this is not a possibility. Thus a robust method for dealing with air-water systems is of great interest, and while we have no definitive answer to this question in this paper, we shall discuss the capabilities of our methods in this respect.

One important feature of the study of atomizers is the need to study the flow both inside objects with complex geometries such as injector nozzles, and over a large range of scales, from the small scale ligaments that form at the nozzle exit to the large scale instabilities of the jet's core. Thus it is useful to have at one's disposal both a method to adjust the mesh size to the scale of the details being studied and to be able to represent complex objects in the simulation. 
Both capabilities are present in the Gerris code which uses a cut-cell method to represent the solid objects and adaptive octree mesh refinement. Gerris is Open Source and freely available at (Popinet, 2008).

In this paper we shall give a number of preliminary simulations of the kind of complex atomizing flows that can be obtained using that type of code. We start with a description of the method, then continue with a comparison of the results of the code with temporal linear theory. We then show various types of flows relating to single jet and co-flowing atomizer devices and study how perturbations of the interface grow spatially. Finally we show some more complex examples involving complex atomizer shapes and swirl atomizers.

\section{Numerical scheme}

The numerical scheme has been described in detail by Popinet (2003, 2007). The following sections give a summary of the main techniques used to obtain an accurate adaptive solution of the incompressible, variable-density, NavierStokes equations with surface tension.

\subsection{Temporal discretisation}

The incompressible, variable-density, Navier-Stokes equations with surface tension can be written

$$
\begin{gathered}
\rho\left(\partial_{t} \boldsymbol{u}+\boldsymbol{u} \cdot \boldsymbol{\nabla} \boldsymbol{u}\right)=-\boldsymbol{\nabla} p+\boldsymbol{\nabla} \cdot(2 \mu \boldsymbol{D})+\sigma \kappa \delta_{s} \boldsymbol{n}, \\
\partial_{t} \rho+\boldsymbol{\nabla} \cdot(\rho \boldsymbol{u})=0 \\
\boldsymbol{\nabla} \cdot \boldsymbol{u}=0
\end{gathered}
$$

with $\boldsymbol{u}=(u, v, w)$ the fluid velocity, $\rho \equiv \rho(\boldsymbol{x}, t)$ the fluid density, $\mu \equiv \mu(\boldsymbol{x}, t)$ the dynamic viscosity and $\boldsymbol{D}$ the deformation tensor defined as $D_{\mathrm{ij}} \equiv\left(\partial_{i} u_{j}+\right.$ $\left.\partial_{j} u_{i}\right) / 2$. The Dirac distribution function $\delta_{s}$ expresses the fact that the surface tension term is concentrated on the interface; $\sigma$ is the surface tension coefficient, $\kappa$ and $\boldsymbol{n}$ the curvature and normal to the interface.

For two-phase flows we introduce the volume fraction $c(\boldsymbol{x}, t)$ of the first fluid and define the density and viscosity as

$$
\begin{aligned}
& \rho(c) \equiv c \rho_{1}+(1-c) \rho_{2}, \\
& \mu(c) \equiv c \mu_{1}+(1-c) \mu_{2},
\end{aligned}
$$

with $\rho_{1}, \rho_{2}$ and $\mu_{1}, \mu_{2}$ the densities and viscosities of the first and second fluids respectively. The advection equation for the density can then be replaced with an equivalent advection equation for the volume fraction

$$
\partial_{t} c+\nabla \cdot(c \boldsymbol{u})=0
$$


A staggered in time discretisation of the volume-fraction/density and pressure leads to the following formally second-order accurate time discretisation

$$
\begin{gathered}
\rho_{n+\frac{1}{2}}\left[\frac{\boldsymbol{u}_{n+1}-\boldsymbol{u}_{n}}{\Delta t}+\boldsymbol{u}_{n+\frac{1}{2}} \cdot \nabla \boldsymbol{u}_{n+\frac{1}{2}}\right]=-\nabla p_{n+\frac{1}{2}}+\nabla \cdot\left[\mu_{n+\frac{1}{2}}\left(\boldsymbol{D}_{n}+\boldsymbol{D}_{n+1}\right)\right]+\left(\sigma \kappa \delta_{s} \boldsymbol{n}\right)_{n+\frac{1}{2}} \\
\frac{c_{n+\frac{1}{2}}-c_{n-\frac{1}{2}}}{\Delta t}+\nabla \cdot\left(c_{n} \boldsymbol{u}_{n}\right)=0 \\
\boldsymbol{\nabla} \cdot \boldsymbol{u}_{n}=0
\end{gathered}
$$

This system is further simplified using a classical time-splitting projection method (Chorin, 1969)

$$
\begin{gathered}
\rho_{n+\frac{1}{2}}\left[\frac{\boldsymbol{u}_{\star}-\boldsymbol{u}_{n}}{\Delta t}+\boldsymbol{u}_{n+\frac{1}{2}} \cdot \boldsymbol{\nabla} \boldsymbol{u}_{n+\frac{1}{2}}\right]=\boldsymbol{\nabla} \cdot\left[\mu_{n+\frac{1}{2}}\left(\boldsymbol{D}_{n}+\boldsymbol{D}_{\star}\right)\right]+\left(\sigma \kappa \delta_{s} \boldsymbol{n}\right)_{n+\frac{1}{2}},(1) \\
\frac{c_{n+\frac{1}{2}}-c_{n-\frac{1}{2}}}{\Delta t}+\nabla \cdot\left(c_{n} \boldsymbol{u}_{n}\right)=0 \\
\boldsymbol{u}_{n+1}=\boldsymbol{u}_{\star}-\frac{\Delta t}{\rho_{n+\frac{1}{2}}} \nabla p_{n+\frac{1}{2}} \\
\boldsymbol{\nabla} \cdot \boldsymbol{u}_{n+1}=0
\end{gathered}
$$

which requires the solution of the Poisson equation

$$
\boldsymbol{\nabla} \cdot\left[\frac{\Delta t}{\rho_{n+\frac{1}{2}}} \nabla p_{n+\frac{1}{2}}\right]=\nabla \cdot \boldsymbol{u}_{\star}
$$

This equation is solved efficiently using the quad/octree-based multilevel solver described in detail in Popinet (2003).

The discretised momentum equation (11) can be re-organised as

$\frac{\rho_{n+\frac{1}{2}}}{\Delta t} \boldsymbol{u}_{\star}-\nabla \cdot\left[\mu_{n+\frac{1}{2}} \boldsymbol{D}_{\star}\right]=\nabla \cdot\left[\mu_{n+\frac{1}{2}} \boldsymbol{D}_{n}\right]+\left(\sigma \kappa \delta_{s} \boldsymbol{n}\right)_{n+\frac{1}{2}}+\rho_{n+\frac{1}{2}}\left[\frac{\boldsymbol{u}_{n}}{\Delta t}-\boldsymbol{u}_{n+\frac{1}{2}} \cdot \nabla \boldsymbol{u}_{n+\frac{1}{2}}\right]$

where the right-hand-side depends only on values at time $n$ and $n+1 / 2$. This is an Helmholtz-type equation which can be solved efficiently using a variant of the multilevel Poisson solver. The resulting Crank-Nicholson discretisation of the viscous terms is formally second-order accurate and unconditionally stable.

The velocity advection term $\boldsymbol{u}_{n+\frac{1}{2}} \cdot \nabla \boldsymbol{u}_{n+\frac{1}{2}}$ is estimated using the BellColella-Glaz second-order unsplit upwind scheme (Bell et al., 1989; Popinet, 2003). This scheme is stable for CFL numbers smaller than one.

\subsection{Spatial discretisation}

Space is discretised using an octree. We refer the reader to Popinet (2003) and references therein for a more detailed presentation of this data structure. All the variables (components of the momentum, pressure and passive tracers) are collocated at the centre of each cubic discretisation volume. Consistently with a finite-volume formulation, the variables are interpreted as the volume-averaged values for the corresponding discretisation volume. The choice of a collocated definition of all variables makes momentum conservation simpler when dealing 
with mesh adaptation (Popinet, 2003). It is also a necessary choice in order to use the Godunov momentum advection scheme of Bell et al. (1989), and it simplifies the implementation of the Crank-Nicholson discretisation of the viscous terms; however one has to be careful to avoid the classic problem of decoupling of the pressure and velocity field.

To do so, an approximate projection method (Almgren et al., 2000; Popinet, 2003 ) is used for the spatial discretisation of the pressure correction equation (3) and the associated divergence in the Poisson equation. In a first step the auxiliary cell-centred velocity field $\boldsymbol{u}_{\star}^{c}$ is computed using equation (5). An auxiliary face-centred velocity field $\boldsymbol{u}_{\star}^{f}$ is then computed using averaging of the cell-centred values on all the faces of the Cartesian discretisation volumes. When faces are at the boundary between different levels of refinement of the quad/octree mesh, averaging is performed so as to guarantee consistency of the corresponding volume fluxes (see Popinet (2003) for details).

The divergence of the auxiliary velocity field appearing on the right-handside of equation (4) is then computed for each control volume as the finite-volume approximation

$$
\boldsymbol{\nabla} \cdot \boldsymbol{u}_{\star}=\frac{1}{\Delta} \sum_{f} \boldsymbol{u}_{\star}^{f} \cdot \boldsymbol{n}^{f},
$$

with $\boldsymbol{n}^{f}$ the unit normal vector to the face and $\Delta$ the length scale of the control volume.

After solving equation (4), the pressure correction is applied to the facecentred auxiliary field

$$
\boldsymbol{u}_{n+1}^{f}=\boldsymbol{u}_{\star}^{f}-\frac{\Delta t}{\rho\left(c_{n+\frac{1}{2}}^{f}\right)} \nabla^{f} p_{n+\frac{1}{2}},
$$

where $c_{n+1 / 2}^{f}$ is obtained by averaging from the cell-centred values $c_{n+1 / 2}^{c}$ and $\nabla^{f}$ is a simple face-centred gradient operator (consistent at coarse/fine volume boundaries (Popinet, 2003)). The resulting face-centred velocity field $\boldsymbol{u}_{n+1}^{f}$ is exactly non-divergent by construction.

The cell-centred velocity field at time $n+1$ is obtained by applying a cellcentred pressure correction

$$
\boldsymbol{u}_{n+1}^{c}=\boldsymbol{u}_{\star}^{c}-\left|\frac{\Delta t}{\rho\left(c_{n+\frac{1}{2}}^{f}\right)} \nabla^{f} p_{n+\frac{1}{2}}\right|^{c},
$$

where the $\|^{c}$ operator denotes averaging over all the faces delimiting the control volume. The resulting cell-centred velocity field $\boldsymbol{u}_{n+1}^{c}$ is approximately divergence-free.

\subsection{Volume-of-Fluid advection scheme}

To solve the advection equation (2) for the volume fraction we use a piecewiselinear geometrical Volume-of-Fluid (VOF) scheme (Scardovelli and Zaleski, 1999) 
generalised for the quad/octree spatial discretisation. Geometrical vof schemes classically proceed in two steps:

1. Interface reconstruction

2. Geometrical flux computation and interface advection

Interface reconstruction is performed using a piecewise-planar interface representation in each cell defined by equation

$$
\boldsymbol{m} \cdot \boldsymbol{x}=\alpha .
$$

Given the interface normal $\boldsymbol{m}$ and the volume fraction $c$ in a given cell, $\alpha$ can be determined uniquely using analytical relations (Guevffier et al., 1998; Scardovelli and Zaleski, 2001). The interface normal $\boldsymbol{m}$ can be approximated by considering the volume fractions in a neighborhood of the cell considered. We use the Mixed-Youngs-Centred (MYC) implementation of Aulisa et al. (2007) on a $3 \times 3 \times 3$ stencil generalised for the octree spatial discretisation (Popinet, 2007).

Once interface reconstruction has been performed, direction-split geometrical fluxes can be computed easily on regular Cartesian grids (DeBar, 1974; Noh and Woodward, 1976; Li, 1995). As shown by Popinet (2007) this is also true for octree spatial discretisations provided some care is taken when dealing with fluxes across coarse-fine discretisation boundaries.

The resulting advection scheme preserves sharp interfaces and has been shown to be close to second-order accurate for practical applications (Aulisa et al., 2007). While this scheme is not strictly conservative (Rider and Kothe, 1998), total conservation errors for difficult problems are usually less than $0.01 \%$.

\subsection{Balanced-force surface tension calculation}

The accurate estimation of the surface tension term $\left(\sigma \kappa \delta_{s} \boldsymbol{n}\right)_{n+\frac{1}{2}}$ in the discretised momentum equation (11) has proven one of the most difficult aspects of the application of vOF methods to surface-tension-driven flows. The original Continuum-Surface-Force (CSF) approach of Brackbill et al. (1992) is known to suffer from problematic parasitic currents when applied to the case of a stationary droplet in theoretical equilibrium (Lafaurie et al., 1994; Popinet and Zaleski, 1999; Harvie et al., 2006). Other methods based on a phase-field description of the interface suffer from similar problems including level-sets and front-tracking with distributed surface-tension.

Recently Renardv and Renardv (2002) and Francois et al. (2006) noted that in the case of a stationary droplet parasitic currents are due to the imbalance between the numerical discretisations of the surface tension and of the corresponding pressure gradient. They also showed that this imbalance can be rectified by using compatible discretisations of the pressure and volume fraction gradients used in the CSF approximation, provided the exact value of the interface curvature is known. 
Computing an accurate estimate of the curvature from the discrete volume fraction values is non-trivial. Renardy and Renardy showed that accurate estimates can be obtained with their parabola-fitting technique but at great computational cost. Cummins et al. (2005) showed that the Height-Function (HF) method initially proposed by Torrev et al. (1985) gives second-order accurate curvature estimates while being much cheaper than parabola-fitting, however Francois et al. (2006) concluded that it was still not accurate enough to solve the problem of parasitic currents around a stationary droplet.

In contrast one of us has shown recently Popinet (2007) that the combination of a balanced-force surface tension discretisation and a Height-Function curvature estimation is sufficient to solve the problem of parasitic currents, provided the initial non-equilibrium interface shape is allowed enough time to relax to its equilibrium shape. This relaxation occurs on a timescale comparable to the viscous dissipation timescale as expected from physical considerations. Furthermore, the numerical equilibrium shape was shown to converge at second-order rate toward the exact equilibrium shape.

The Height-Function technique is relatively simple to extend to an octree spatial discretisation but may become inconsistent when the radius of curvature of the interface is less than approximately five times the grid spacing (Popinet, 2007). In these cases the paraboloid fitting technique of Popinet (2007) is used. The transition between the two curvature estimation techniques has been shown to be consistent with overall second-order accuracy. The increased robustness provided by this approach is important when dealing with the sprays formed during jet atomization.

\subsection{Mesh adaptation}

The overall scheme allows for space and time-varying spatial resolution. To simplify the implementation the sizes of neighboring cells can not vary by more than a factor of two (this is sometimes referred to as restricted octree). While this can limit the efficiency of adaptation for three-dimensional problems which have a fractal dimension close to two (Min and Gibou, 2007), this should not be an issue for most complex fluid dynamics problems, including atomization.

In contrast to many previous implementations of mesh adaptation for interfacial flows with Eulerian discretisations ((Jeong and Yang, 1998: Sochnikov and Efrima, 2003: Wang et al., 2004; Greaves, 2004; Kohno and Tanahashi, 2004; Theodorakakos and Bergeles, 2004; Zheng et al., 2005; Greaves, 2006; Nikolopoulos et al., 2007; Min and Gibou, 2007) with the exception of Malik et al. (2007)) our method is not limited to constant resolution along the interface. This can dramatically increase the efficiency of mesh adaptation, particularly when dealing with reconnections and breakup of interfaces (Popinet, 2007).

One of the advantages of the octree disctretisation is that mesh refinement or coarsening are cheap and can be performed at every time-step if necessary with 
a minimal impact on overall performance Interpolation of quantities on newly refined or coarsened cells is also relatively simple and is done conservatively both for momentum and volume fraction (Popinet, 2007).

Several refinement criteria can be used simultaneously depending on the problem. Combinations of the following criteria have been used in this article:

\section{Vorticity}

$$
\frac{|\boldsymbol{\nabla} \times \boldsymbol{u}| \Delta}{\max (|\boldsymbol{u}|)}<\epsilon,
$$

with $\Delta$ the mesh size and $\epsilon$ a user-defined threshold. This criterion will ensure that a finer resolution is used in areas of high vorticity. The threshold parameter $\epsilon$ can be interpreted as the maximum angular deviation from a straight path - due to the local vorticity $\boldsymbol{\nabla} \times \boldsymbol{u}$ - of a massless particle travelling at speed $\max (|\boldsymbol{u}|)$ across a cell of size $\Delta$. The threshold $\epsilon$ is usually set to a small value, typically $10^{-2}$. For interfacial flows, vorticity generation is often associated with high interface curvatures. Using this criterion will thus also ensure that small radii of curvature are properly resolved.

\section{Gradient}

$$
|\nabla c| \Delta<\epsilon,
$$

with $c$ a field variable. This criterion provides for example a simple way to create a "band" of constant high resolution around an interface defined by volume fraction $c$. With a second order discretisation of $\nabla c,|\nabla c|$ will be non-zero only in a band of approximately three cells around any cell cut by the interface. Setting $\epsilon$ to a very small value and putting an upper bound on the maximum level of refinement will ensure that the cells cut by the interface and their immediate neighbours always use the highest level of refinement.

\section{Curvature}

$$
\kappa \Delta<\epsilon,
$$

with $\kappa$ the curvature of the interface if the cell contains a fragment of interface, zero otherwise. By construction $1 / \epsilon$ is the minimum number of cells required to discretise a given radius of interface curvature. It is typically set to 0.2 (i.e. five cells per radius of curvature). In constrast to the gradient criterion, the curvature criterion will result in a variable resolution along the interface when the interface curvature varies. This means for example that smooth, planar interfacial sheets can be resolved using a coarse resolution, while small, localised "cusps", bubbles and droplets use higher resolution. As demonstrated in Popinet (2007) this can lead to orders-of-magnitude savings in simulation size. 


\section{Results}

\subsection{Comparison with linear theory}

The experimental validation of the numerical solutions obtained for twophase flows presents several problems. From the experimental point of view, there is a high uncertainty of the measurements in atomization experiments. On the other hand, various numerical issues appear when two-phase flows with large density and viscosity ratios are simulated at high Reynolds numbers.

In this work, the numerical code is validated using the temporal viscous Linear Theory (LT) applied to two-phase parallel mixing layers. This theory predicts the temporal growth of the small disturbances induced in the flow and for that reason it is an ideal framework for measuring the performance of the code for capturing the behavior of the instabilities in real situations.

The Linear Theory predicts the temporal response to a small perturbation $\phi_{l, g} e^{i \alpha(x-c t)}$ imposed on a given base flow. The solution of the temporal growth rate is given by the Orr-Sommerfield equations, which derive from the NavierStokes equations:

$$
\begin{aligned}
\left(U_{l}-c\right)\left(D^{2}-\alpha^{2}\right) \phi_{l}-D^{2} U_{l} \phi_{l} & =\frac{1}{i \alpha R e_{l}}\left(D^{2}-\alpha^{2}\right)^{2} \phi_{l}, \\
\left(U_{g}-c\right)\left(D^{2}-\alpha^{2}\right) \phi_{g}-D^{2} U_{g} \phi_{g} & =\frac{m}{r} \frac{1}{i \alpha R e_{l}}\left(D^{2}-\alpha^{2}\right)^{2} \phi_{g} .
\end{aligned}
$$

with $r=\rho_{g} / \rho_{l}$ the density ratio and $D$ denotes the derivative with respect to y.

To solve these equations, some boundary conditions must be imposed. In particular, a rectangular domain problem is considered here, where $-L_{l}<y<0$ for the liquid phase and $0<y<L_{g}$ for the gas phase.

The initial velocities profiles are imposed (Figure 1)

$$
\begin{aligned}
& U_{l}(y)=U_{i n t}-U_{l}^{*} \operatorname{erf}\left(y / \delta_{l}\right) \\
& U_{g}(y)=U_{i n t}+U_{g}^{*} \operatorname{erf}\left(y / \delta_{g}\right)
\end{aligned}
$$

$U_{l}^{*}$ being the liquid velocity, $U_{g}^{*}$ the gas velocity (* denotes the absolute values far from the interface) and $\delta_{g}$ and $\delta_{l}$ are the thickness of the gas and liquid boundary layer respectively. Velocities are defined respective to a system of reference moving at the interface velocity, that is $U_{\text {int }}=0$. The velocities far away from the interface are related through the stress continuity condition:

$$
\frac{U_{g}^{*}}{U_{l}^{*}}=\frac{n}{m}
$$

where $m=\mu_{g} / \mu_{l}$ is the viscosity ratio and $n=\delta_{g} / \delta_{l}$ is the ratio between the boundary layer thicknesses. Further details about the method of resolution of 


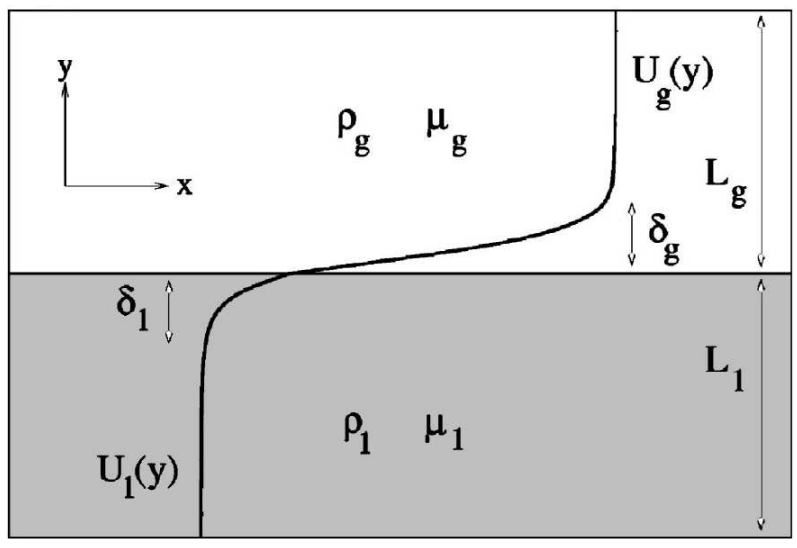

Figure 1: Parameters and profiles used for the liquid and gas phases.

Eq. (9) can be found in Yecko et al. (2002).

In order to interpret the results it is useful to define the following Reynolds and Weber numbers, whose values determine the type of observed instabilities (Boeck et al., 2007):

$$
\begin{gathered}
R e_{l}^{*}=\rho_{l} U_{l}^{*} \delta_{l} / \mu_{l}, \quad R e_{g}^{*}=\rho_{g} U_{g}^{*} \delta_{g} / \mu_{g} \\
W e_{l}^{*}=\rho_{l}\left(U_{l}^{*}\right)^{2} \delta_{l} / \sigma, \quad W e_{g}^{*}=\rho_{g}\left(U_{g}^{*}\right)^{2} \delta_{g} / \sigma
\end{gathered}
$$

In order to avoid any dependence of the results with this the height of the domain, $L=L_{l}+L_{g}$, it should be satisfied that $L_{g} / \delta_{g} \gg 1, L_{l} / \delta_{l} \gg 1$ and $2 \pi / \alpha<<L_{g}, L_{l}$.

Table 1: Simulation parameters. Effective uniform grid resolution: 256x768

\begin{tabular}{ccccccccc}
\hline reference & $m$ & $r$ & $\delta_{l} / L_{l}$ & $\delta_{g} / L_{g}$ & $R e_{l}^{*}$ & $R e_{g}^{*}$ & $W e_{l}^{*}$ & $W e_{g}^{*}$ \\
\hline $\mathrm{A}$ & 0.1 & 1 & $1 / 6$ & $1 / 6$ & 20 & 2000 & $\infty$ & $\infty$ \\
$\mathrm{B}$ & 0.1 & 1 & $1 / 6$ & $1 / 6$ & 20 & 2000 & 0.1 & 10 \\
$\mathrm{C}$ & 0.99 & 0.1 & $1 / 6$ & $1 / 6$ & 19602 & 2000 & $\infty$ & $\infty$ \\
$\mathrm{D}$ & 0.99 & 0.1 & $1 / 6$ & $1 / 6$ & 19602 & 2000 & 1 & 10 \\
\hline
\end{tabular}

The parameters of Table 1 are used here for validation of the code.

Initially, the flow is perturbed using the eigenfunctions $\phi_{l}$ and $\phi_{g}$ obtained from the solution of the Orr-Sommerfield equations (9)-(10) using Tchebychev polynomials. Thus, the initial state is defined as

$$
u(t=0, x, y)=U(y)+\varepsilon\left(D \phi_{r}(y) \cos (\alpha x)-D \phi_{i}(y) \sin (\alpha x)\right)
$$




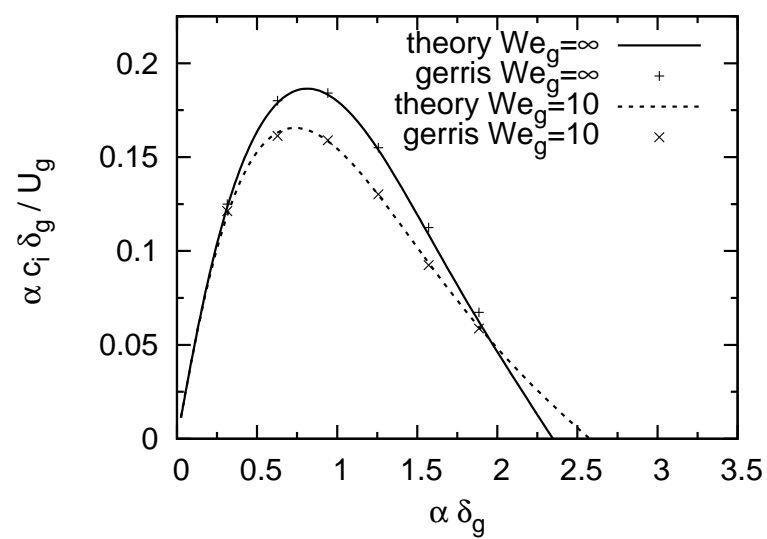

Figure 2: Growth rates of unstable modes and the corresponding results with the Gerris code for cases A and B of Table 1

$$
\left.v(t=0, x, y)=\alpha \varepsilon x\left(\phi_{i}(y) \cos (\alpha x)+\phi_{r}(y) \sin (\alpha x)\right)\right)
$$

where $U(y)$ is the base flow given by Eq. (11), $\varepsilon$ is the amplitude of the initial perturbation and $\phi_{r}(y)$ and $\phi_{i}(y)$ are the real and imaginary parts of the eigenfunction $\phi(y)$.

In the linear regime the apparent growth rate is constant. Just when the instability is large enough the regime becomes non-linear and the apparent growth rate changes. Its measurement is done by fitting the temporal evolution of the amplitude of the main mode in the linear regime, which typically lasts until the amplitude of the perturbation reaches values around $10^{-2} \alpha^{-1}$. The position of the maximum height of the wave has been shown to be enough to provide a good estimation of the growth rate, although the results can be slightly improved by fitting the interface position to the Fourier mode.

The results for cases A and $\mathrm{B}(m=0.1$ and $r=1)$ are depicted in Figure 2 Good agreement is found between numerical and theoretical results. The errors obtained from the comparison between the numerical and theoretical results are always below $5 \%$ either with or without surface tension.

Figure 3] shows the numerical and theoretical curves when $m=0.99$ and $r=0.1$ (cases $\mathrm{C}$ and $\mathrm{D}$ ). In this case, the growth rate $\alpha c_{i}$ predicted by the linear theory perfectly fits the results obtained numerically, the mean errors being always inferior to $1 \%$ with and without surface tension respectively.

For all the test cases, the results converge with the mesh size (Table 2), obtaining error below $5 \%$ for meshes bigger than $128 \times 128$.

Thus, it can be concluded that the solutions provided by the numerical 


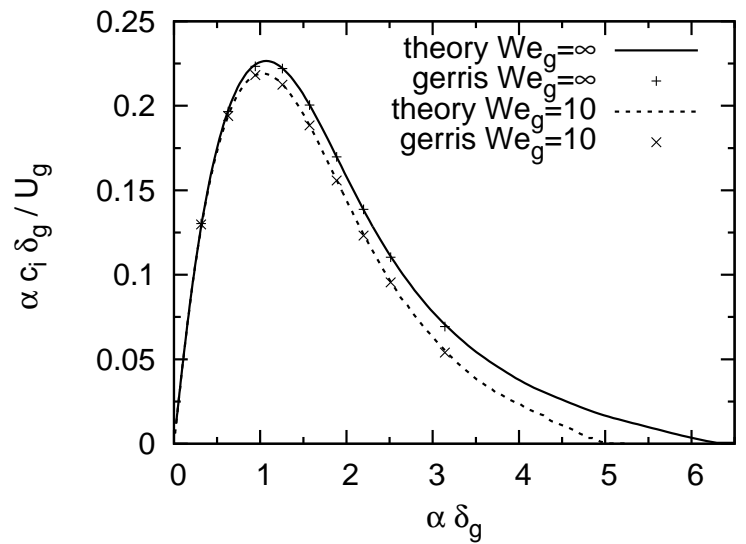

Figure 3: Growth rate of unstable modes and the corresponding results with the Gerris code for cases $\mathrm{C}$ and $\mathrm{D}$ of Table 1

\begin{tabular}{ccccc}
\hline reference & 32 & 64 & 128 & 256 \\
\hline $\mathrm{A}$ & 21.33 & 10.74 & 3.50 & 1.5 \\
$\mathrm{~B}$ & 7.30 & 1.28 & 0.48 & 1.04 \\
$\mathrm{C}$ & 1.17 & 0.24 & 0.14 & 0.09 \\
$\mathrm{D}$ & 1.39 & 0.76 & 0.07 & 0.54 \\
\hline
\end{tabular}

Table 2: Error percentage for the different cases from Table 1 Mesh sizes: 32x32, 64x64, $128 \times 128$ and $256 \times 256$.

code are in a good agreement with the theoretical solution obtained from the Orr-Sommerfeld equation. The results are especially accurate for viscosity and density ratios near one. Nevertheless, differences are still acceptable when the density ratio is decreased. As a conclusion it can be considered that, for the range of conditions used in this work, the code provides reliable solutions for the analysis of the instabilities in free shear flows.

\subsection{Study of the instabilities produced in primary atomization}

After validating the code, the next step is to try to analyze relatively simple problems which can shed some light on the real physical processes taking place in primary atomization. The simulations included in this part of the paper aim at capturing the real behavior of the jet during the first stage of the injection, where instabilities are produced and amplified before the jet is broken into droplets. The study of the frequencies and wavelengths encountered in the primary atomization, together with the amplitude of these disturbances should influence the final droplet distribution and other features of the spray. For that reason, it is crucial to clarify the source of these disturbances and to understand the physical processes which can influence their amplification. 
The influence of the turbulence model on the obtained results, already studied by Ménard et al. (2005, 2007), have awaken great interest in Direct Numerical Simulations (DNS). However, the DNS simulation of multiphase flows is not a simple task. To ensure that all the length scales of the problem are captured, extremely refined meshes are required. Apart from the turbulent scales, small structures like thin sheets or very small droplets can appear, significantly increasing the computational time usually associated to DNS in single phase flows.

The use of accurate adaptive numerical schemes allows concentration of the computational effort in zones where the small scales are present. This feature, together with the the balanced-force surface tension calculation implemented in Gerris (Popinet, 2007), has allowed to significantly reduce the computational time required for well-resolved simulations. Two criterias have been used to perform the refinement: The vorticity and the gradient of the tracer variable (both with $\epsilon=2.5 \cdot 10^{-3}$ ). The efficiency of the mesh adaptation strongly depends on the considered problem. For the simulations included in this section the reduction in terms of number of cells due to AMR is around $61 \%$ for the two-dimensional simulations whereas for the $3 \mathrm{D}$ example this value increases up to $95 \%$. These values can be significantly improved as we enlarge the simulation domain and also the resolution. In more complex problems where large simulation domains must be simulated with a large resolution just over some localized parts of the domain, the number of cells required to perform the simulation with AMR can be several orders of magnitude less than the number of cells which would be required if an uniform grid were used.

The efficiency of AMR, $\eta_{\mathrm{AMR}}$, can be defined as

$$
\eta_{\mathrm{AMR}}=\frac{t_{\text {uniform }} / \mathrm{Size}_{\text {uniform }}}{\mathrm{t}_{\mathrm{AMR}} / \mathrm{Size}_{\mathrm{AMR}}}
$$

where $t_{\text {uniform }}$ is the time invested to perform a given number of steps using an

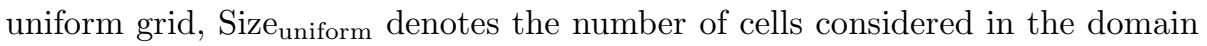
and the subscript AMR is used to specify that the values are calculated using AMR. $\eta_{\mathrm{AMR}}$ is 1 when the percentage of time saved due to AMR is equal to the percentage of reduction of the number of cells. Again, it is difficult to define characteristic values of $\eta_{\mathrm{AMR}}$ as they significantly depends on the problem. Just an example, the efficiencies in two-dimensional and three-dimensional simulations have reached values around 0.8 .

For all problems the tolerance of the pressure solver is set to $10^{-4}$. The stopping criterion is $\left\|a R_{l}\right\|_{\infty}<10^{-4}$, where a is the fraction of fluid contained in the cell and $R_{l}$ is the residual computed at the deepest level. In any case, long computational times are still required and the parallelization of these problems is mandatory. Just as an example, for the two dimensional simulations shown in this section, the computational time is approximately 48 hours on a 4 processor Dual Core AMD Opteron(tm) 265. For the 3D simulation shown at the end of 
this section, the run time is increased up to 5 days on 25 processors.

The level of refinement used in the simulations is large enough to ensure that the finest scales of the vortices generated in each of the phases are captured. This considerably restricts the Reynolds numbers which can be reached but it ensures the reliability of the results. In any case, it must be clarified that strictly speaking the term of DNS cannot be applied to these simulations. Even if all the scales related to the turbulence are well-resolved, likely some structures such as ligaments and droplets still have a characteristic length smaller than the minimum grid size (as can be observed on the right of Figure 4). Then, for the study of the instabilities encountered in the primary atomization zone, it is assumed that structures smaller than the minimum grid spacing (typically smaller than one micron) have no effect on the waves that appear in this zone.

Finally, certain disturbances have been artificially imposed at the entrance in order to initiate the growth of instabilities. This condition is directly linked with the perturbations which are induced in the flow upstream, although other effects like the stress non-equilibrium at the interface just after the injector or the thickness of the separator plate can also have an influence on the appearance of instabilities. To capture these effects, the simulation of the entire injector would provide the characteristic frequencies of the turbulence generated inside it. However, the introduction of the injector shape in the problem introduces geometrical variables which significantly increases the complexity of the analysis of the results. In most of this work, some ideal case studies are considered where the flow inside the injector is not considered and the disturbances are triggered off by means of perturbations at the entrance. At high Reynolds and Weber numbers, where the solutions turn more intricate, the two-dimensional problem is considered. For low Reynolds, 3D simulations are possible and they have been used to capture some of the effects related appearing in the primary atomization zone.

\subsubsection{Analysis of the instabilities at high Reynolds numbers}

In this section, a high degree of accuracy is obtained using meshes with a smallest mesh size of about $0.8 \mu \mathrm{m}$. Output boundary conditions are applied in all boundaries of the domain except at the entrance, where the velocity profile is prescribed according to Eq. (11) with an interface velocity defined by the stress condition at the interface (Eq. (13)). Moreover, a perturbation $v^{\prime}$ is introduced in the vertical velocity, which is a random function applied either in the liquid or in the gas giving values inside the range $[-0.1 \mathrm{U}: 0.1 \mathrm{U}]$. The rest of conditions required for the simulation are included in Table 3 and the relevant dimensionless parameters for these simulations can be found in Table 4. As initial condition the same base velocity profile (11) is used throughout and the void fraction is set equal to 0 .

Figure 4 is a representative view of the behavior of the jet for the conditions indicated above. As can be seen, the simulation is limited to the zone where 
Table 3: Simulation conditions used for the analysis of the instabilities in the primary atomization zone. Effective uniform grid resolution: 1536x512

\begin{tabular}{ccccccc}
\hline & $U(\mathrm{~m} / \mathrm{s})$ & $\rho\left(\mathrm{kg} / \mathrm{m}^{3}\right)$ & $\mu(\mathrm{Pa} \cdot \mathrm{s})$ & $\sigma(\mathrm{N} / \mathrm{m})$ & Domain size $(\mu \mathrm{m})$ & $\delta(\mu \mathrm{m})$ \\
\hline Liquid & 20 & 1000 & $5 \cdot 10^{-4}$ & 0.03 & 40 & 11.8 \\
Gas & 100 & 200 & $1.7 \cdot 10^{-5}$ & & 60 & 2.0 \\
\hline
\end{tabular}

Table 4: Representative dimensionless numbers for the conditions given in Table 3

\begin{tabular}{ccccccc}
\hline$m$ & $r$ & $R e_{l}^{*}$ & $R e_{g}^{*}$ & $W e_{l}^{*}$ & $W e_{g}^{*}$ & $M$ \\
\hline 0.034 & 0.200 & 471 & 2350 & 157 & 133 & 5.00 \\
\hline
\end{tabular}

the instabilities are induced and propagate before breaking up into droplets.

Two main zones can be distinguished:

- In the first zone, the disturbances at the entrance generate small perturbations which are quickly amplified downstream. Figure 5 depicts the velocity fluctuations at different positions from the entrance. In the very early stage of the injection the initial turbulence induced in the liquid is transferred to the zone near the interface (transition from $x / \delta_{g}=0$ to $\left.x / \delta_{g}=25\right)$. Then, even if the small disturbances introduced at the entrance are quickly dissipated in the bulk of the liquid due to viscous effects, they are strong enough to create some instabilities at the interface.

As the perturbations grow downstream, the level of turbulence inside the gas and liquid increases, mainly in zones near the interface. The small perturbation originally induced in the liquid finally generates a strong turbulence downstream not only in the liquid, but also in the gas. Remarkably, this phenomenon is also observed when the instabilities are induced in the gas (Figure 6). In this case, the gas momentum is large enough to generate appreciable perturbations at the interface, which are finally amplified producing a strong turbulence in both phases.

- In the second zone the instabilities become non-linear and ligaments appear. These ligaments are finally broken into droplets which interact with the vortex appearing in the gas phase near the interface. For large Reynolds numbers the flow structures in the gas vortex generated under the ligaments strongly interact with the drops which have been generated. Therefore, simulation results are extremely sensitive to the mesh size and a DNS simulation of the jet is required, restricting considerably the Reynolds numbers which can be reached with a high degree of accuracy. 


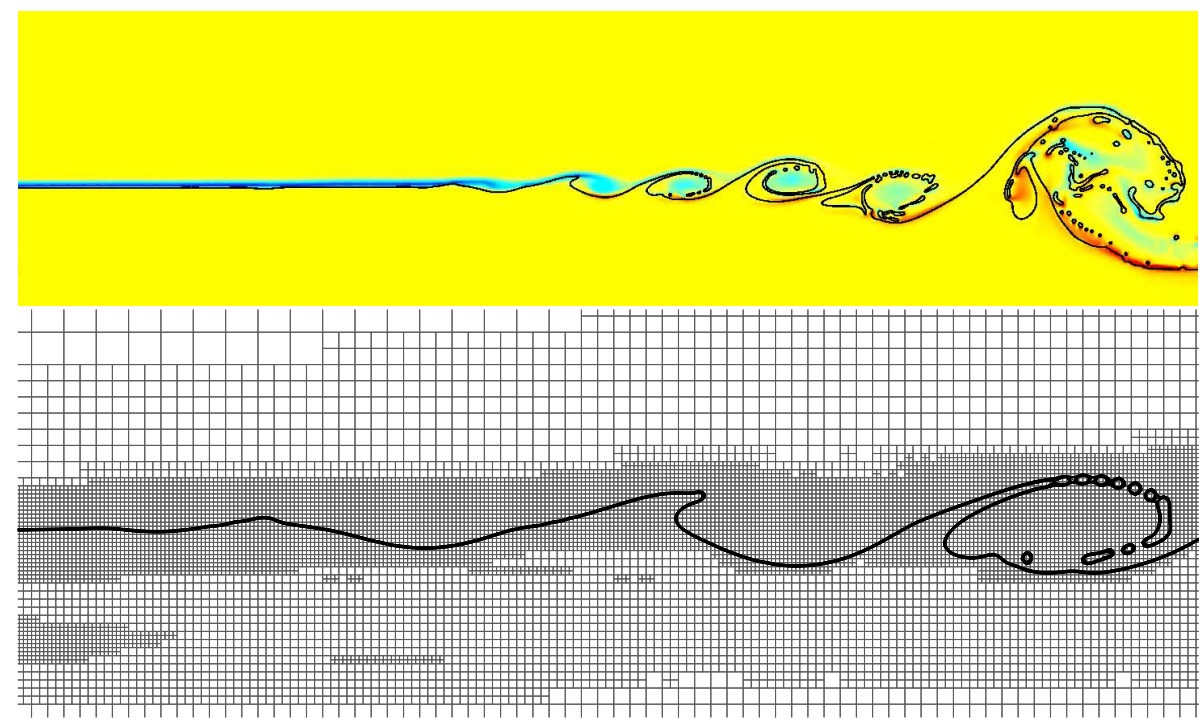

Figure 4: Interface profile along a distance of $400 \mu \mathrm{m}$ downstream of the injector at $t=50 \mu \mathrm{s}$ (top). Zoom on the transition between the linear and non-linear regime (bottom). Minimum mesh size: $0.2 \mu \mathrm{m}$.
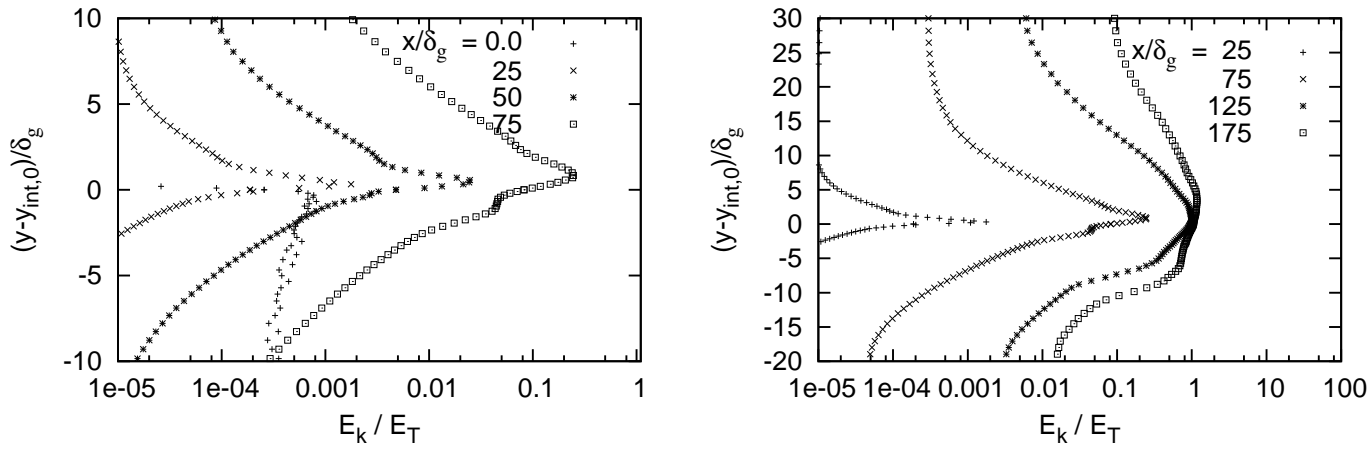

Figure 5: Profiles of the velocity fluctuations $\left(E_{k}=\left(u_{i}^{\prime}\right)^{2}\right)$ normalized with the input velocity $\left(E_{T}=(U(x=0))^{2}\right)$ at different distances from the injector. Perturbation is imposed only in the liquid phase. 


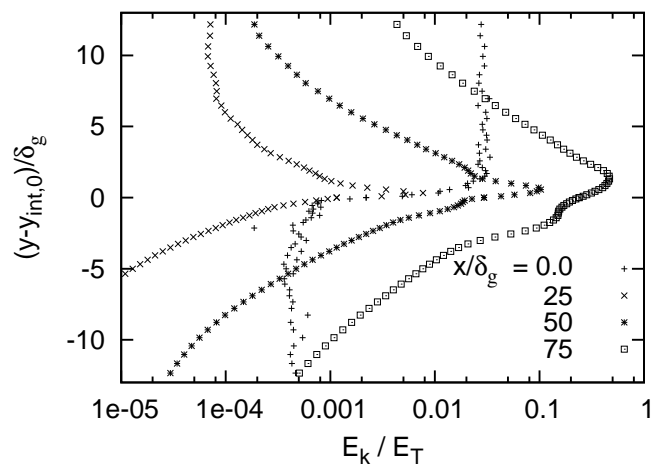

Figure 6: Profiles of the velocity fluctuations $\left(E_{k}=1 / 2\left(u_{i}^{\prime}\right)^{2}\right)$ at different distances of the injector. The perturbation is solely induced in the gas phase.

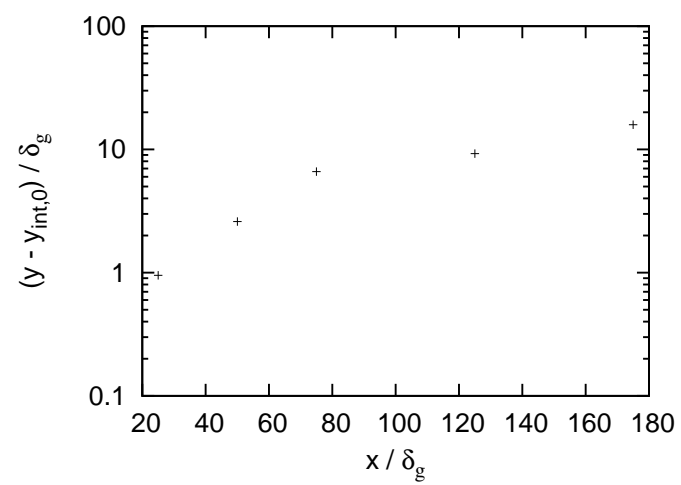

Figure 7: Evolution of the average amplitude of the waves along the jet. Transition from a linear regime with an exponential growth of the waves $\left(x / \delta_{g} \leq 80\right)$ to the non-linear regime.

To conclude, the turbulence near the inflow boundary in any of the both phases is promoting the appearance of instabilities at the interface, whose amplification is responsible for the transition from the linear to the non-linear regime. This transition is captured in Figure 7 where the time-averaged maximum of the interface position at different locations is plotted as a function of the distance from the injector. In the first zone $\left(x / \delta_{g} \leq 80\right)$, a clear exponential growth is observed. Once the amplitude of the waves is much larger than the thickness of the boundary layer, the growth rate saturates.

In the linear regime, the initial growth rate is a function of parameters such as the Reynolds and Weber numbers or the gas and viscosity ratios. Some attempts have been made using linear theory to predict the characteristic frequencies which are amplified downstream; however the lack of a full theory for the spatial problem and the difficulties related with the temporal change of the 
base flow makes numerical simulations compulsory in order to investigate this type of situations. Nevertheless, it is interesting to compare some of the results obtained from simulations with those obtained from the linear theory presented in section 3.1

Firstly, it is important to emphasize that numerical results are compared with theoretical results based on the assumption that the instability is convective. This property is revealed by stopping the perturbation at the inlet. For the case studied here, the waves are then propagated downstream and the equilibrium state given by the input boundary conditions is recovered.

Another effect which should be taken into account is the evolution of the base flow downstream. The changes in the velocity profiles as well as those in the boundary layer thickness can alter the behavior of the different modes. Figure 8] depicts different velocity profiles at different positions downstream of the input boundary condition. Just near to the entrance the velocity profile is similar to that introduced as an input boundary condition. The boundary layer then grows due to viscous effects and when the flow becomes non-linear (for distances larger than $x / \delta_{g}=80$ ) the velocity profile is significantly modified due to the turbulence generated in the mixing boundary layer.
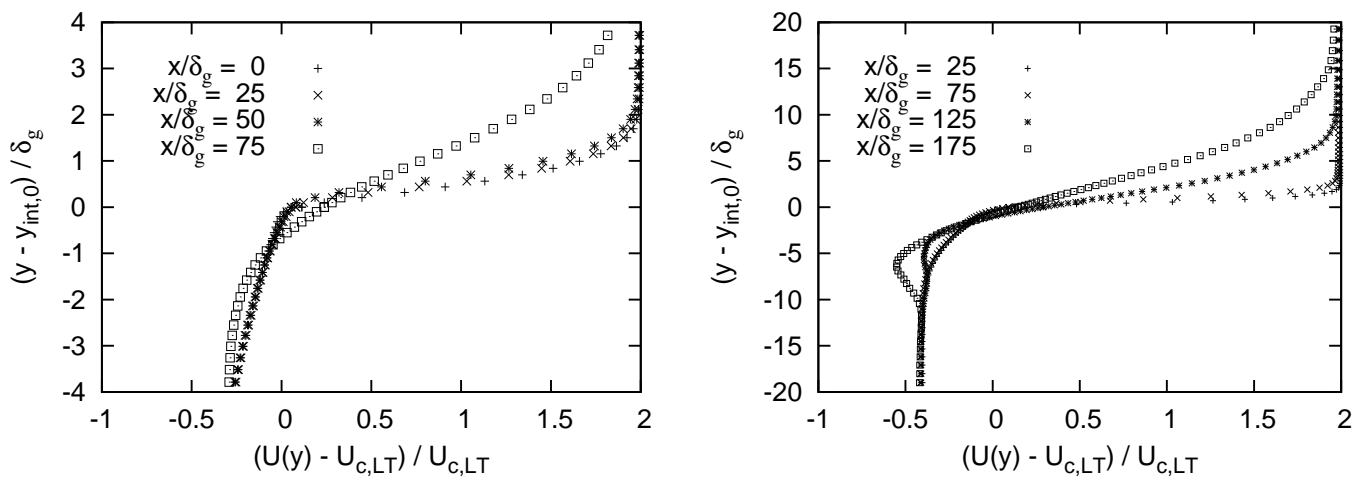

Figure 8: Evolution of the velocity profile in different sections. Left graph: Evolution of the velocity profile in the zone where waves are still linear. Right: Transition from the linear to the non-linear zone.

In a first approximation, the results obtained by the temporal stability analysis with the input velocity profile are compared with the simulations during the first moments. Figure 9 depicts the growthrates predicted by the viscous linear theory. The inviscid linear theory is also shown as it has been extensively used by some authors to discuss experimental results (Ben Ravana et al., 2006; Marmottant and Villermaux, 2004). Although in many cases inviscid and viscous predictions differ (Boeck and Zaleski, 2005) in the present case the pre- 


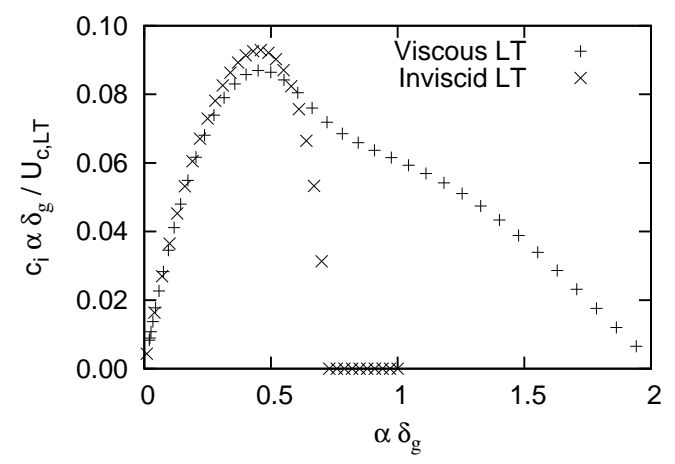

Figure 9: Growth rates predicted by the temporal inviscid and viscous linear theory.

dictions are similar. All the wavelengths satisfying $\alpha \delta_{g}<2$ are expected to be amplified, with an optimum growthrate for a dimensionless wavelength near 0.5.

The theoretical results are compared with the numerical ones by means of the analysis of the spatial-temporal signal of the interface position. The signal is analyzed after some time, when the flow reaches a quasi-steady state and the initial transient effects are negligible.

The frequencies are obtained applying the Fourier Transform method (FT) to the signal of the interface position at a given location $x$. The continuous representation of the spectrum power for every $x$ provides the spectrogram, which gives information about the evolution of the frequencies along the jet (Figure [10).

At the entrance, the random perturbation generates a background noise that excites all the frequencies. Downstream, these disturbances are amplified at different rates. No resonant frequencies are found and a continuous spectra is obtained which seems to be in agreement with a convective instability. A zoom on the non-linear regime reveals the predominance of small frequencies which can be attributed to the vortex pairing in two phase mixing boundary layers.

For a more direct comparison between simulation and theoretical results, four spectra at different locations are plotted in Figure 11 The interpretation of the results has to be understood under the following assumptions:

- The instability being identified as convective, it is considered that some convective velocity relates the frequencies measured in the simulations, $f$, and the wavelengths predicted by theory $\alpha^{-1}$. As a first approximation this velocity is taken as constant and equal to the velocity of the interface introduced as an input boundary condition.

$$
U_{c, L T}=\frac{f}{\alpha}
$$




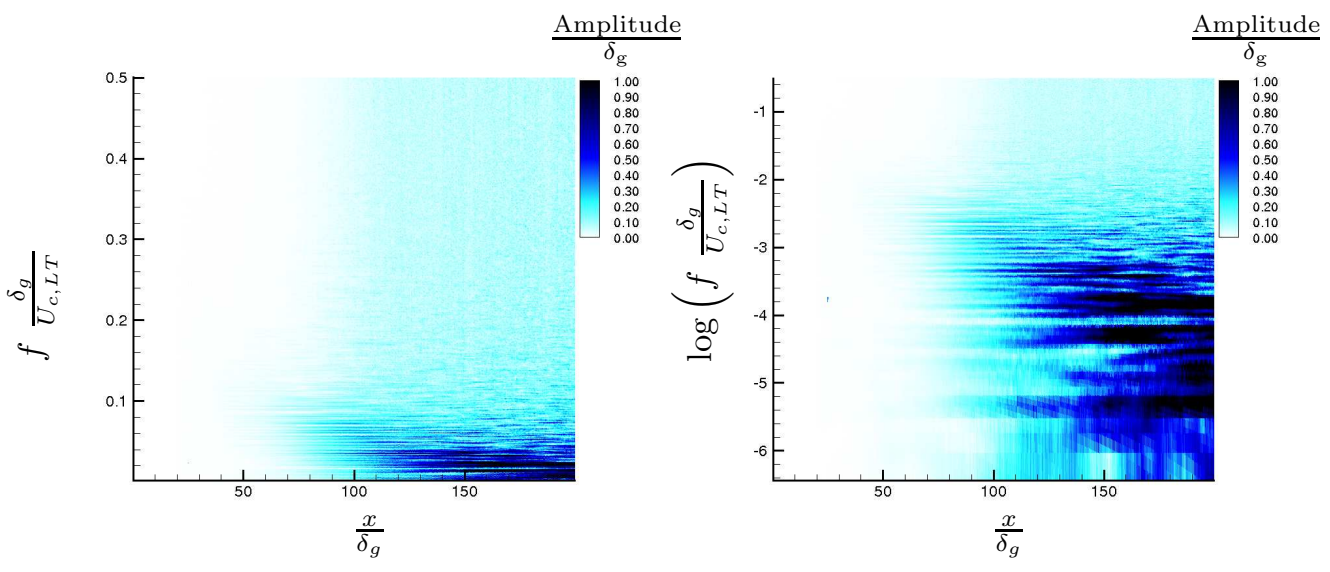

Figure 10: Spectrogram of the interface position: Amplitude and frequency of the temporal interface oscillations at every position. Homogeneous noise is introduced at the entrance.

- The initial perturbation amplitude is assumed to be equal for all the frequencies. Thus, the amplitude at some distance $x$ from the injector would be proportional to the growthrate $c_{i}$.

Despite the simplifications done to perform the comparison, a good agreement is observed between the numerical and theoretical spectra. Results fit especially well for the first spectrum $\left(x / \delta_{g}=25\right)$, where the perturbations are small enough so that linear stability theory may hold. As explained, the most amplified frequencies tend to decrease downstream which is a consequence of the growth of the vortices in the two phase mixing boundary layer. When the instability is large enough, the vortices generated in the gas phase predominately excite low frequencies which become even smaller as the phenomenon of vortex pairing becomes important downstream.

The trends obtained from the frequency analysis can also be extracted from the wavelengths. In this case, we decided to extract the FT of the spatial signal applied to different domain sizes (Figure 12). Again, the most amplified frequency decreases as the flow evolves downstream.

\subsubsection{Analysis of the instabilities at low Reynolds numbers}

For low Reynolds numbers the flow patterns near the injector are significantly less complex, allowing to perform 3D simulations with a degree of accuracy good enough to capture the flow patterns near the injector. In the examples shown in this section, constant velocities are imposed as input boundary conditions 

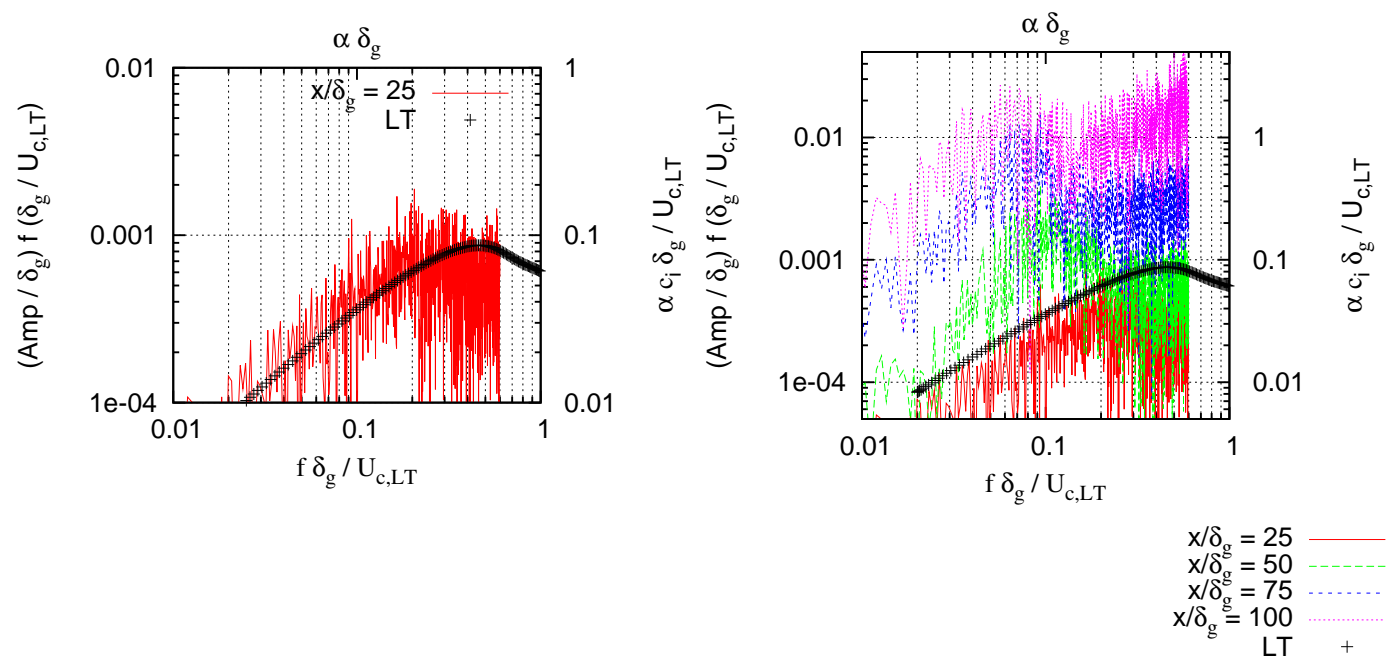

Figure 11: FT of the temporal signal at different locations (lines) and theoretical growthrate (dots).

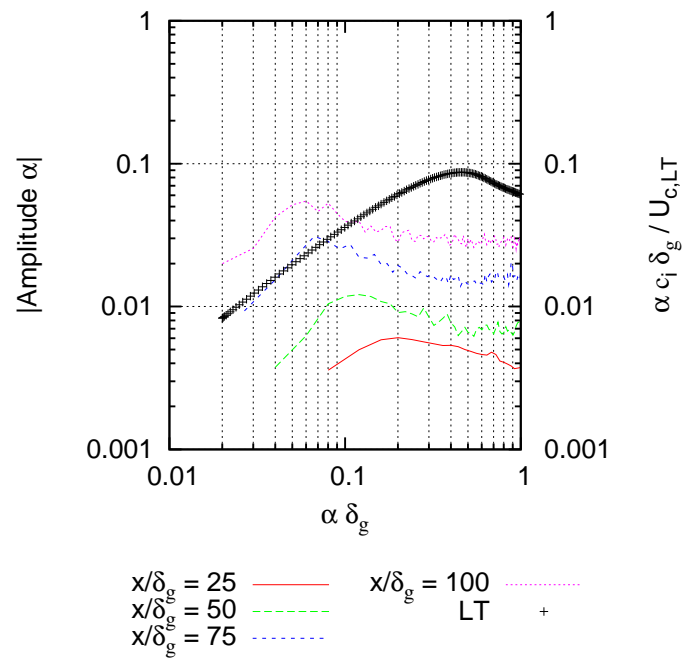

Figure 12: Evolution of the spatial growthrate of the waves measured by means of the FT of the spatial signal using different spatial windows. The amplification of the waves downstream as well as the decrease in the dominant wavelengths as the measurement point moves is apparent from the figure.

in both phases, liquid and gas. Therefore, no boundary layer thickness can be defined. Instead, the thickness of the separator plate $(e)$ is introduced as a 


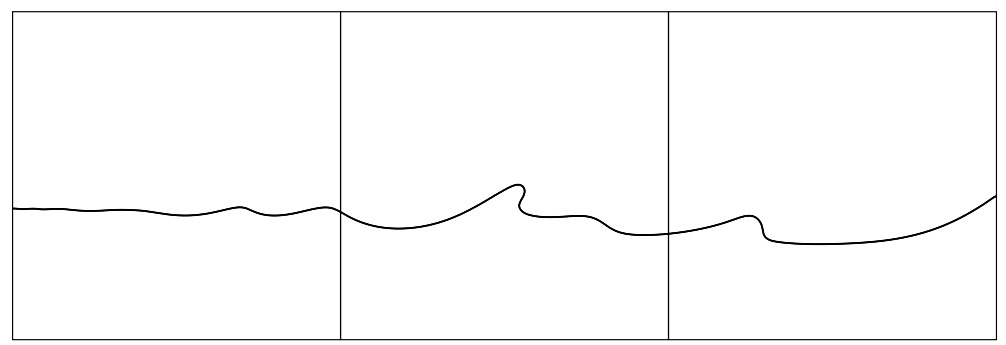

Figure 13: Two-dimensional simulation. Interface representation. Some instabilities are generated an amplified downstream. Due to the low Reynold number, the amplitude of the waves is smaller than those obtained at higher Reynolds numbers.

characteristic distance. The conditions used to perform this simulation are included in Table 5 and the characteristic dimensionless parameters are contained in Table 6

Table 5: Parameters for the simulations at low Reynolds numbers. Effective uniform grid resolution: $512 \times 128 \times 128$ in $3 \mathrm{D}$ and $512 \times 128$ in $2 \mathrm{D}$

\begin{tabular}{ccccccc}
\hline & $U(\mathrm{~m} / \mathrm{s})$ & $\rho\left(\mathrm{kg} / \mathrm{m}^{3}\right)$ & $\mu(\mathrm{Pa} \cdot \mathrm{s})$ & $\sigma(\mathrm{N} / \mathrm{m})$ & $R_{\text {inj }}(\mathrm{m})$ & $e(\mu \mathrm{m})$ \\
\hline Liquid & 20 & 20 & $2 \cdot 10^{-3}$ & 0.03 & $4 \cdot 10^{-4}$ & 80 \\
Gas & 100 & 2 & $1 \cdot 10^{-4}$ & & $6 \cdot 10^{-4}$ & 80 \\
\hline
\end{tabular}

Table 6: Representative dimensionless numbers for the conditions given in Table 5

\begin{tabular}{ccccccc}
\hline$m$ & $r$ & $R e_{l}^{*}$ & $R e_{g}^{*}$ & $W e_{l}^{*}$ & $W e_{g}^{*}$ & $M$ \\
\hline 0.005 & 0.100 & 80 & 145 & 48.5 & 19.4 & 5.00 \\
\hline
\end{tabular}

The flow patterns obtained from the two-dimensional simulation (Figure 13) are not significantly different from those obtained from the 3D simulation of the coaxial flow (Figure 14). Note also that the instability is still triggered by means of a random perturbation in the two-dimensional simulation whereas no turbulence at the entrance is artificially introduced in the 3D simulation. In this case, the presence of a solid plate and the velocity disequilibrium is enough to promote the apparition of instabilities along the jet. Once small perturbations of the interface appear, these waves are propagated and amplified downstream. In this region, 3D effects become important and lead to non axi-symmetric behavior.

Thus, the 3D simulation allows to capture the transverse instabilities which cannot be captured by two-dimensional models; however, in the zone nearest to the injectior, no three-dimensional effects are observed and the two-dimensional 


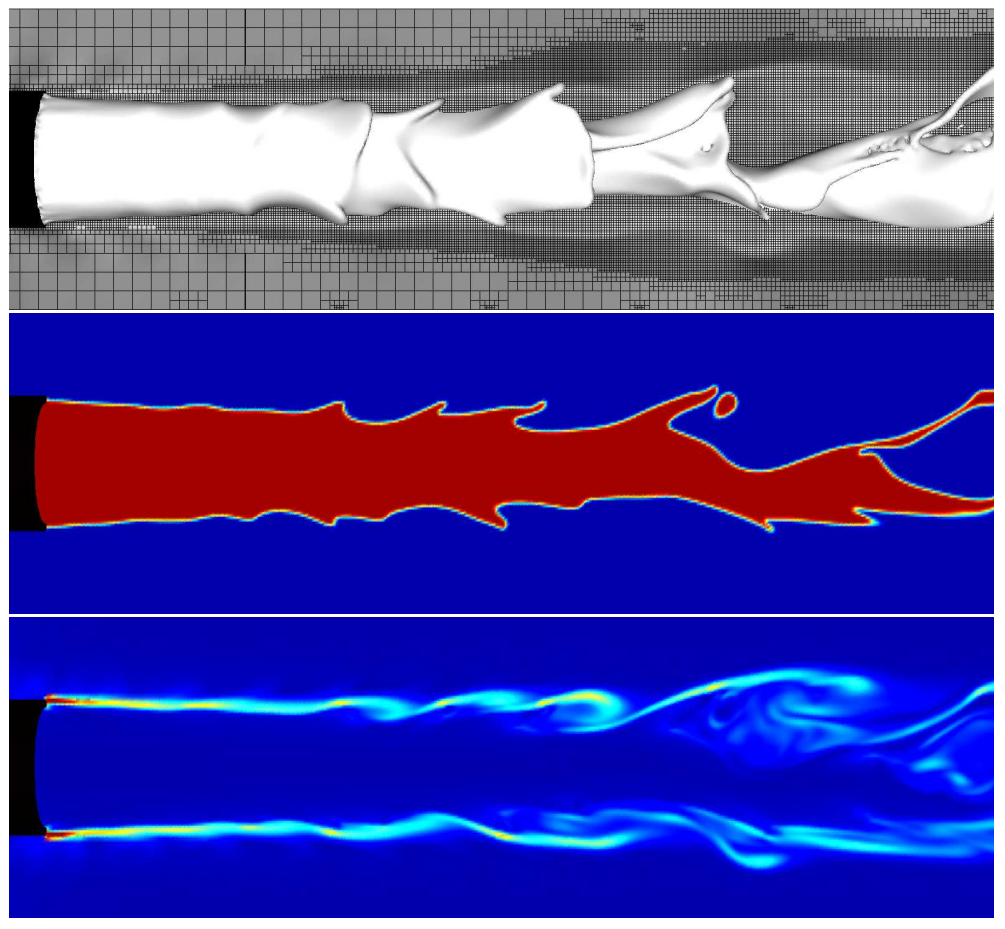

Figure 14: Three-dimensional simulation. Interface representation, liquid fraction and vorticity profiles in a median plane. 


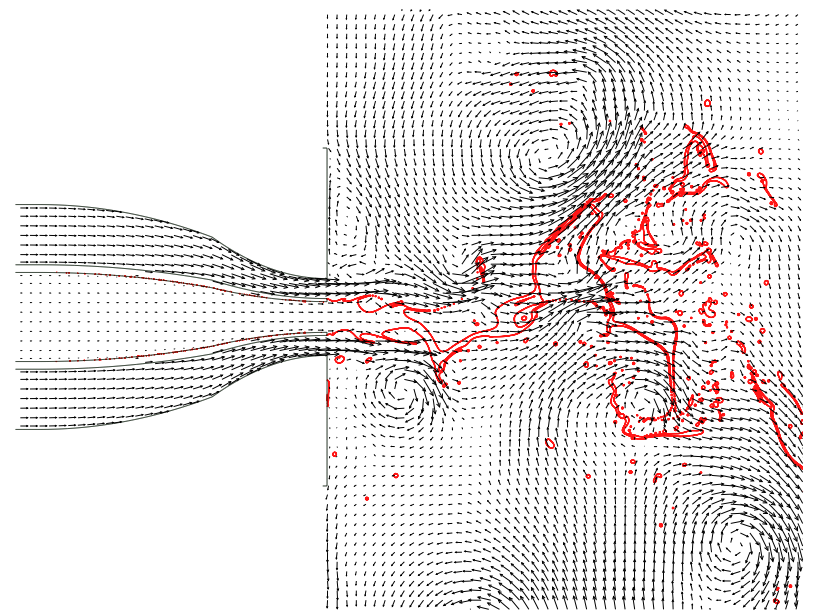

Figure 15: Two-dimensional simulation of the flow inside the injector and the injection chamber. Intense and extended gas vortices are generated which interact with the liquid jet.

simulation seem to correctly capture the mechanisms controlling wave amplification.

In real situations, the flow inside the injector and the evolution of the vortices generated inside the injector chamber can also have a significant influence on the flow patterns observed. The inclusion of the injector significantly increases the computational resources required to perform the simulation. Even for relatively simple situations like those shown in this work, large computational resources are required to perform the simulation of the entire geometry. In Figures [15. 16] the results obtained from the two-dimensional simulation of the injector are depicted. The conditions are set in order to have the same conditions at the entrance of the chamber than those of previous simulations (Table 5 ).

The inclusion of the injector and the chamber downstream makes possible the capture of the gas vortices which strongly interact with the liquid jet (Figure 15). This phenomenon, together with the effect of the flow just behind the separator plate (Figure 16), produce relatively large disturbances which significantly alter the flow patterns in comparison with the simplified situations presented before. Here, the potential of the code to study such complex phenomena is only illustrated but much larger efforts should be done in the future in order to understand and characterize the flow in real systems. Nevertheless, current simulations results point out the importance of the thickness of the separator plate and the physical phenomena taking place close to it, therefore, theories related to the wake stability could be helpful for the analysis of the characteristic frequencies observed in this zone (Ho and Huerre, 1984; Oertel, 1990; Roshko, 1954). 


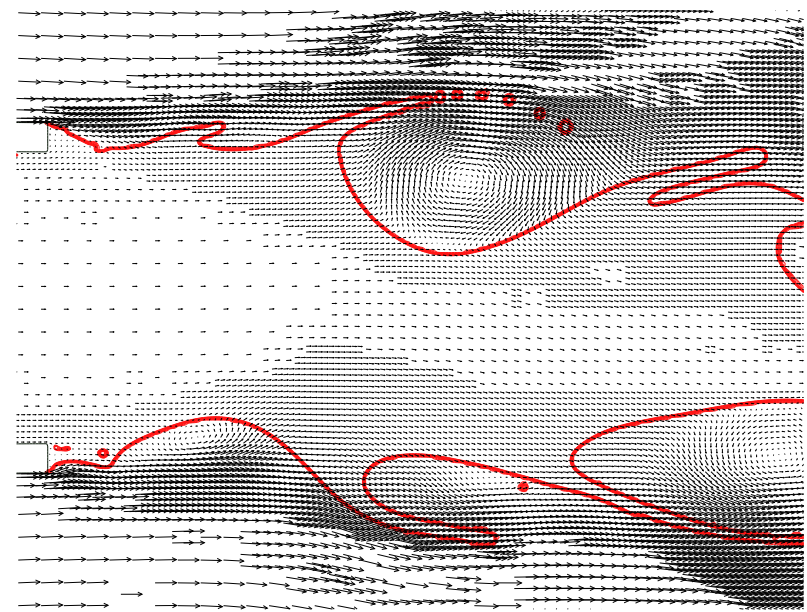

Figure 16: Zoom on the liquid injection zone. The liquid is attached to the external walls of the separator plates. Relatively large instabilities are encountered in this zone which quickly grow and become non-linear.

To sum up, it can be concluded that two-dimensional simulations provide accurate enough information about the behavior of the jet in the linear regime. In this region, the Reynolds and Weber number as well as the ratio of physical variables like density and viscosity strongly influence the type of instabilities observed (Boeck et al., 2007). Some distance downstream, transverse instabilities appear and 3D simulations have to be used to correctly predict the jet behavior. These instabilities can appear both in the linear regime (Yecko and Zaleski, 2005) and in the non-linear regime. Different theories have been proposed in the literature to explain the main mechanisms leading to 3D instabilities, however, little numerical and experimental evidences have been given supporting any of these theories (Ben Ravana et al., 2006; Marmottant and Villermaux, 2004; Yecko and Zaleski, 1999). As shown in this paper, the development of the numerical techniques makes possible the capture of transverse instabilities which should provide in a near future new insights into the main mechanisms governing the formation of 3D ligaments and sheets.

Regarding numerical issues, we have found that the mesh size is critical in order to capture all the droplet sizes and vortex scales of the flow, significantly restricting the achievable Reynolds numbers. In the next section, a more detailed analysis is presented to investigate how sensitive to the mesh size some parameters of the simulation are.

\subsection{Simulation of complex jets}

Among the different types of atomizers typically used in combustion chambers, swirl outward-opening jets (also called hollow-cone atomizers) are particularly interesting due to their complex flow features. Usually encountered in 
injectors designed for viscous liquids in a steady atmosphere, the main characteristics of these injectors are the axial and azimuthal velocities transfered to the jet. At high enough levels of rotational velocity, the breaking mechanisms in the jet are enhanced and the formation of droplets is promoted.

The theoretical analysis of these problems turns out to be extremely complicated and it is necessary to resort to numerical simulations for a correct understanding of the jet behavior. In any case, some authors have addressed the problem of annular sheets for medium curvature ranges and inviscid flows. Applying stability theory, it is possible to predict the maximal amplification of surface disturbances through a dispersion relation which links the wave amplification with the spatial wave number. Some authors like Senecal et al. (1999) have proposed simplified versions of this equation for providing upperbound for the estimation of the wavelengths, but the complexity of the problem impedes the development of accurate theoretical models able to predict the spray behavior. Just recently, with the aid of a numerical treatment, the whole swirling annular linearized flow has been addressed by Jeandel and Dumouchel (1999) and Sekar (2005), who have solved the fourth-order dispersion equation dependent on axial and azimuthal wave numbers.

For the particular case of swirl atomizers, linear theory has an inherent limited framework of validity and when considering the relatively high complexity of its results concerning swirling flows, the necessity of accurate simulations of this kind of atomizers becomes evident. Thus, in this section, a study of closeto-reality swirling atomizers is presented as an example of the potential of the code to tackle complex multiphase problems.

The main parameter controlling the performance of these equipments is the swirl velocity. The initial dimensionless velocity profile in the simulations is :

$$
\begin{aligned}
& U_{x}=1 \quad \text { if } \quad \mathrm{y}^{2}+\mathrm{z}^{2} \leq \mathrm{R}_{\mathrm{inj}}^{2} \\
& U_{x}=0 \quad \text { if } \quad \mathrm{y}^{2}+\mathrm{z}^{2}>\mathrm{R}_{\mathrm{inj}}^{2} \\
& U_{y}=\alpha \sin [\arctan (z / y)] \\
& U_{z}=\alpha \cos [\arctan (z / y)]
\end{aligned}
$$

and the swirl Weber number is

$$
W e=\frac{f\left(\rho U_{x}^{2} R_{\mathrm{inj}}\right)}{\sigma} \approx 10^{3}
$$

$R_{\text {inj }}$ being the initial liquid radius $(0.2 \mathrm{~mm})$ and $f$ being the swirl parameter varied from 0 to 1.2. From this range of $f$, it can be concluded that the $U_{y}$ and $U_{z}$ components of velocity vary from 0 to 1.2 times the axial velocity. The density ratio is approximately $1 / 30$ and the axial Reynolds number, defined as $2 R_{\text {inj }} \rho_{l} U_{x} / \mu_{l}$, is fixed at $10^{4}$. These full set of parameters is given in Table 7

In an effort to compare some of the results with experimental conditions, experiments carried out in our group in conditions close to simulations are used 
Table 7: Parameters for the simulations for the swirl atomizer. Effective uniform grid resolution: $768 \times 256 \times 256$

\begin{tabular}{cccccc}
\hline & $U_{x}(\mathrm{~m} / \mathrm{s})$ & $\rho\left(\mathrm{kg} / \mathrm{m}^{3}\right)$ & $\mu(\mathrm{Pa} \cdot \mathrm{s})$ & $\sigma(\mathrm{N} / \mathrm{m})$ & $R_{\text {inj }}(\mathrm{m})$ \\
\hline Liquid & 20 & 1000 & $8 \cdot 10^{-3}$ & 0.432 & $2 \cdot 10^{-4}$ \\
Gas & 0 & 35.92 & $2.87 \cdot 10^{-4}$ & & \\
\hline
\end{tabular}

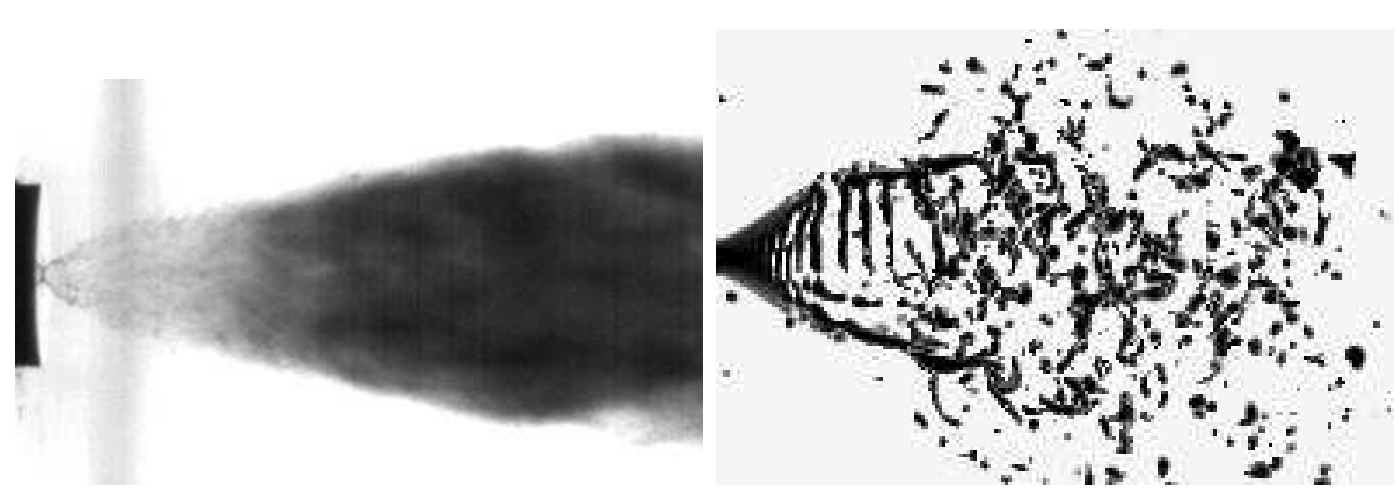

Figure 17: Left: Photography of an automotive injection of iso-octane at 10Mpa injection pressure, $0.1 \mathrm{MPa}$ ambient pressure and hole diameter of approximately $0.3 \mathrm{~mm}$ obtained in Le Movne et al. (2007). Right: Simulation carried out at similar conditions $\left(R e=10^{4}\right.$, $\left.W e=10^{3}\right)$.

(Tröger, 2004). Only the density ratio, 700/5 in experiments and 30/1 in simulations, is markerdly different.

Figure 17 compares two snapshots obtained from experiments (left) and the numerical simulation (right) for a spray generated by a swirling jet. Similar flow patterns are observed for direct image comparison, mainly in the zones near the injection where the characteristic length scales are not smaller than the mesh size yet. General features of atomization such as the breakup length are well represented and the presence of an abrupt change of slope within a small distance downstream seems to be captured. In particular, the experimental cone angle is recovered when the swirl factor $f$ has a value of 0.75 .

The flow patterns can be clearly seen in Figure 18, In the first zone, the cone radius increases due to the rotational velocity introduced at the entrance. As in the examples shown in the previous section, some instabilities appear at the interface which are propagated and amplified downstream. When the instability becomes of the order of the liquid-sheet thickness, a quick breakup process is triggered. Figure 19 shows the velocity field in a median plane. A strong turbulence is generated inside the conical section. It is a consequence of the entrainment of the air by the injected liquid. The inner vortices are responsible for complex droplet motion. Strong vortices are generated in the gas phase once 


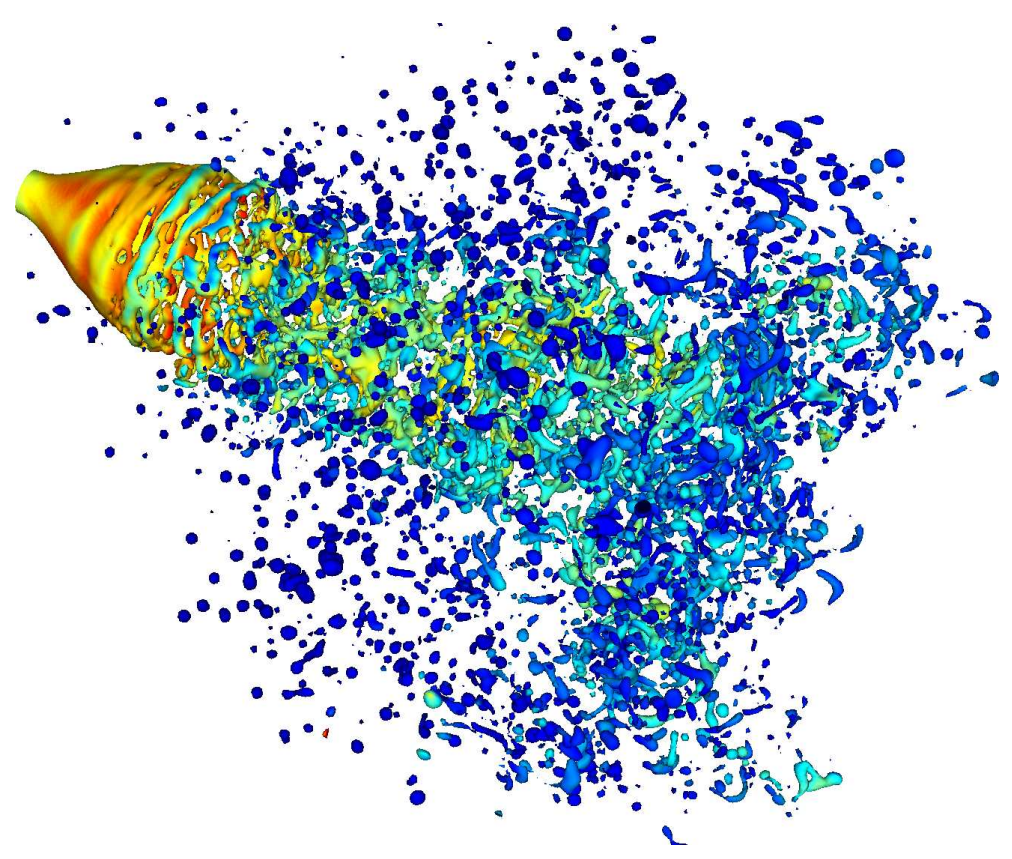

Figure 18: Snapshot of the simulation at $t / t_{0}=3.5$ with a minimum mesh size of $28 \mu \mathrm{m}$. In colors, the velocity norm is represented. A first zone is clearly distinguished where the centrifugal force is responsible for the increase of the cone diameter. Some instabilities are also observed at the surface which are amplified before the jet is definitely disintegrated.

a disperse gas-liquid mixture is obtained, producing a significant change of the slope of the zone occupied by the gas-liquid mixture.

As already explained, when intense turbulence is generated, the mesh size must be small enough to capture the correct physics. The sensitivity of the results with the mesh size is reflected not only in the small structures of the jet (droplets) but also in the large structures. Features of the atomization process are dependent on mesh refinement as can be seen in Figure 20, where different two-dimensional slices from the 3D simulation are shown in a zone close to the injector for three different mesh sizes. As can be seen, both the break-up length and the spray angle are dependent on mesh refinement which the importance of the mesh size. For under-resolved simulations, the droplets and vortices inside the cone are not correctly captured and the physical processes obtained from simulations are unrealistic. In any case, calculations seem to converge to a situation where the change in the initial spray angle is produced in a zone close to the breakup and where the physical mechanisms of the liquid break-up are captured.

Another parameter which is commonly used in computational and experimental analysis for the measurement of the atomization performance is the Probability Density Function (PDF) distribution of the droplet size. In Fig- 


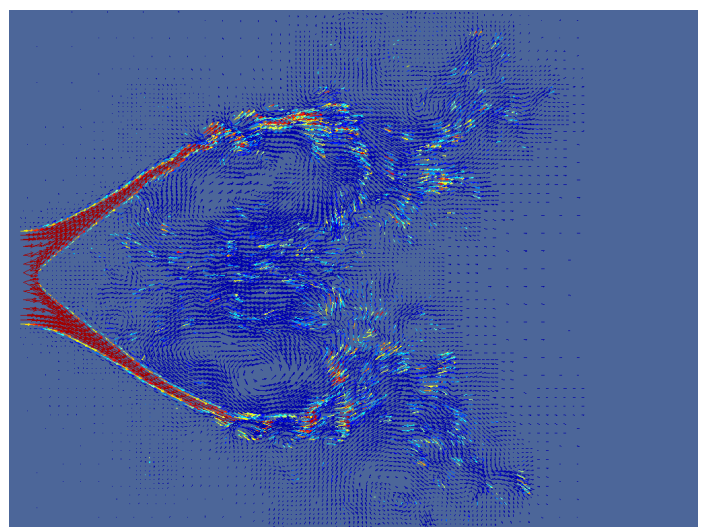

Figure 19: Volume fraction and velocity vector field in a median plane. Minimum mesh size $9 \mu \mathrm{m}$. An empty liquid cone is generated as a consequence of the induced jet rotation. When the instabilities generated in the liquid grow, the liquid sheet is broken and the droplets are dragged along with the turbulent gas phase. At this point, a significant change of slope is observed.
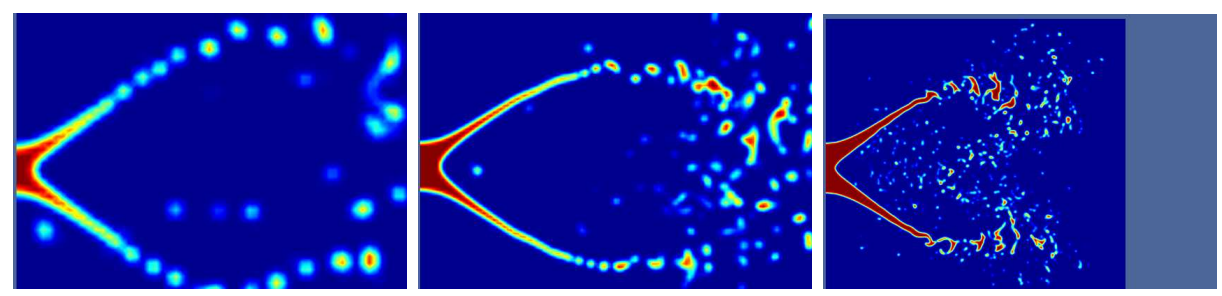

Figure 20: View of volume fraction in a median plane for different mesh sizes (from left to right, $56 \mu \mathrm{m}, 28 \mu \mathrm{m}$ and $9 \mu \mathrm{m}$ ). The influence of the mesh size on the structures captured in the simulations can be observed. Using a coarse mesh, unphysical breakup processes are observed which leads to bigger and unresolved drops and a larger expansion angle. With the finest mesh, the instabilities are captured which leads to a good resolution of the breaking process and a better resolution of the droplets. 

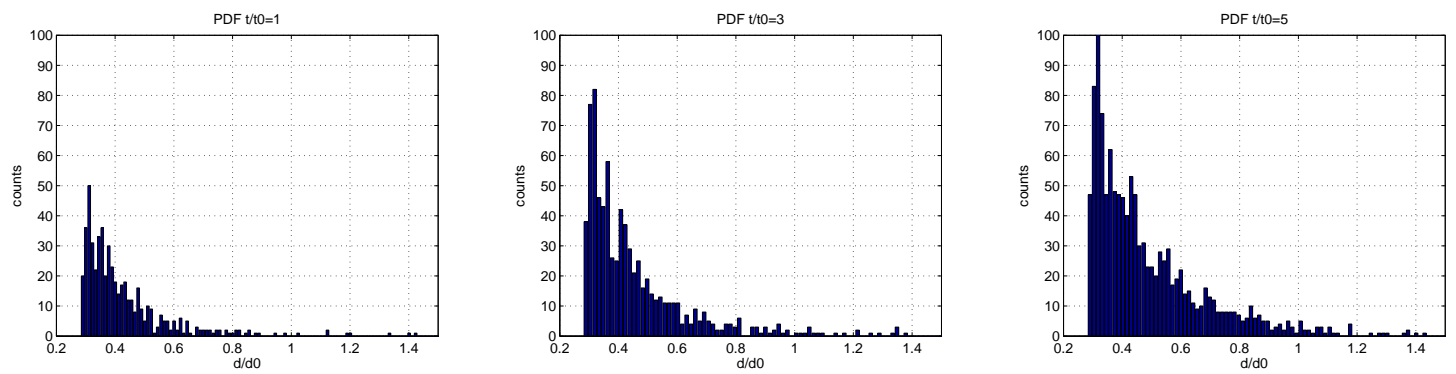

Figure 21: Temporal evolution of the distribution of droplet diameters obtained from simulations non-dimensioned by the initial liquid diameter $d_{0}$.
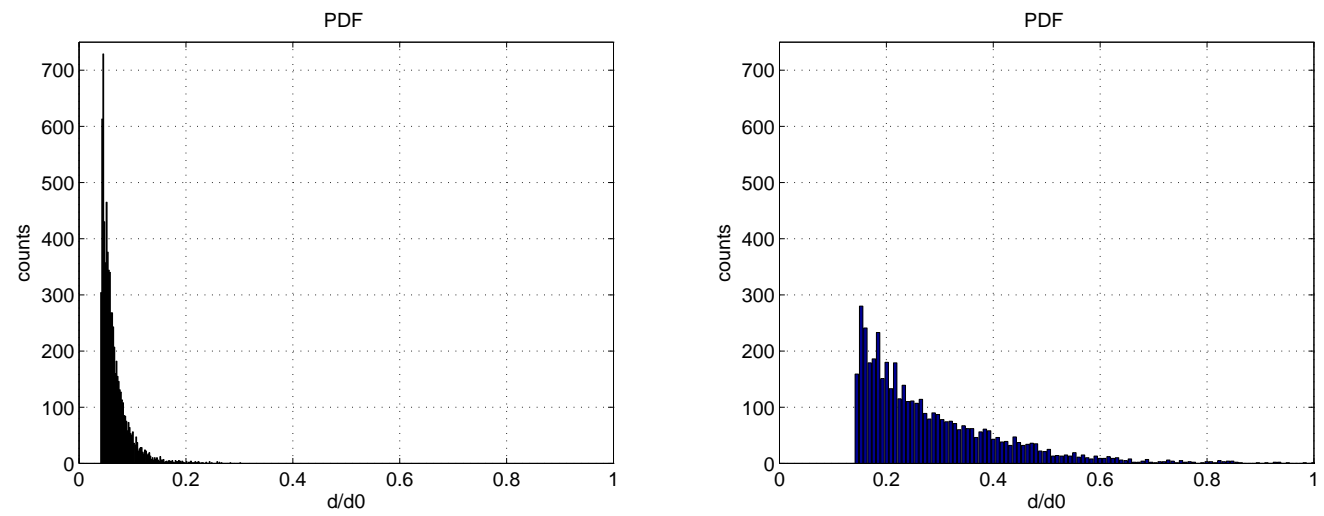

Figure 22: Effect of mesh refinement on PDF distribution. Normalized PDF of droplet diameters non-dimensioned by initial liquid diameter at $t / t 0=3.5$ using a minimum mesh size of $9 \mu \mathrm{m}$ (left) and $28 \mu \mathrm{m}$ (right)

ure 21] the temporal evolution of the PDF is plotted. The droplet diameter is non-dimensioned using the liquid initial diameter $d_{0}$. When mesh refinement is increased, the dimensionless maxima of the PDF distribution decays towards smaller sizes (see Figure 22), resulting for instance in a decrease of the Sauter Mean Diameter $\left(0.7 \cdot d_{0}\right.$ for a mesh of size $56 \mu \mathrm{m}, 0.5 \cdot d_{0}$ for a mesh size of $28 \mu \mathrm{m}$ and $0.1 \cdot d_{0}$ for a mesh of size $\left.9 \mu \mathrm{m}\right)$.

The experimental PDF obtained in Trögen (2004) (Figure 23) fitted by a chi-square distribution of order 15 and maximum diameter $150 \mu \mathrm{m}$, is compared with an order 15 chi-square PDF distribution resulting from our most highlyresolved simulation. As can be observed, despite of the differences due to the different conditions between simulation and experiments, a good agreement for the PDF is obtained when the mesh size is small enough. 

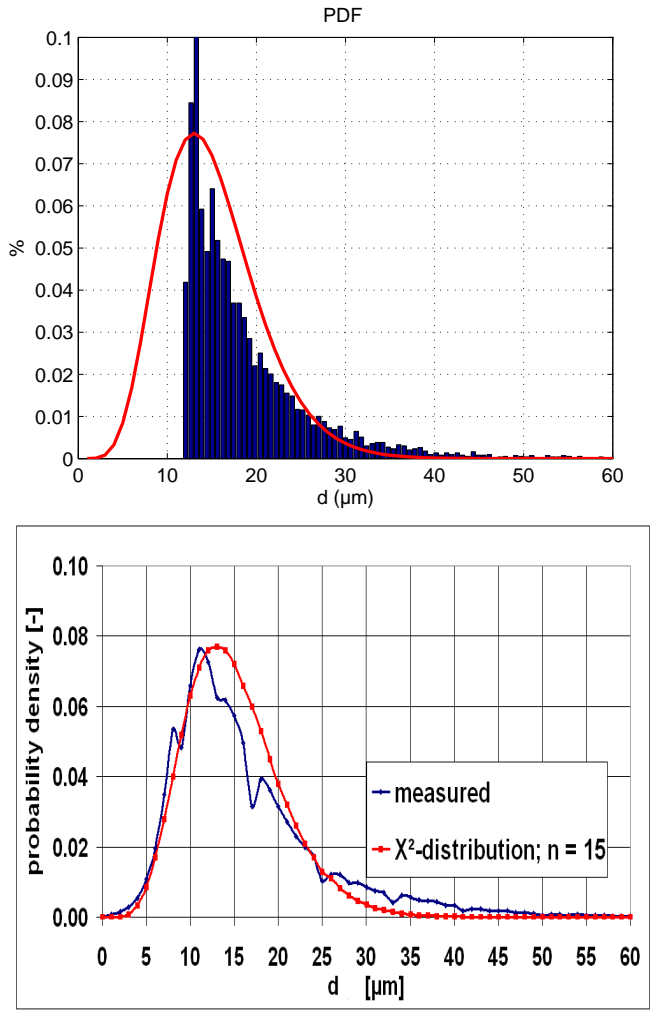

Figure 23: Top: The PDF distribution obtained with a mesh size of $9 \mu \mathrm{m}$ and the chi-square distribution of order 15 and maximum diameter $13 \mu \mathrm{m}$. Below: The experimental PDF obtained in Tröger (2004) and the same chi-square distribution of order 15 and maximum diameter $13 \mu \mathrm{m}$. 


\section{Conclusions}

This paper describes various simulations of primary atomization using an adaptive VOF method. The capabilities of Gerris to perform numerical simulations of two-phase flows have allowed us to investigate the atomization process.

Simulations with characteristic scales spread over several orders of magnitude have been possible using octree adaptive mesh refinement techniques. The spatial resolution is concentrated in places where the solution is more complex, like interfaces and zones of large vorticity, where small cell sizes are required to capture all the flow patterns. Small droplets and vortices are generated and interact strongly. As the Reynolds number increases the flow patterns become more intricate, restricting the Reynolds numbers achievable with an acceptable degree of accuracy. Moreover, 3D effects become more important and the utility of two-dimensional simulations to extract quantitative and qualitative results is more questionable.

The influence of mesh refinement has been tested for swirl atomizers at high Reynolds numbers. For such complex problems numerical simulation is mandatory to understand the jet behavior. For low-resolution simulations, breakup processes and droplet dynamics are unphysical. Using refined meshes the physical mechanisms are correctly captured; some instabilities are initially induced in the jet and are amplified downstream. When the liquid sheet becomes very thin, the movement of the ligaments and droplets is mainly governed by the gas vortices generated inside the liquid cone. At this point, an abrupt change of slope is observed which corresponds with experimental observations in similar conditions (Le Movne et al., 2007).

Finally the capabilities of Gerris to handle gas/liquid/solid interfaces have also allowed us to obtain some preliminary results about the effect of the injector and chamber geometry on the jet behavior. The vortices generated in the injection chamber seem to have a significant influence on the primary atomization process. Other effects like the wakes generated behind the separator plate can also play a role on the initial perturbations induced in the flow. Thus, for a correct understanding of real systems, a simulation of the entire system seems to be crucial in order to understand the interaction of the various physical mechanisms leading to liquid breakup.

As a conclusion, our simulations have illustrated the potential of adaptive and accurate numerical schemes for two-phase flow simulations. The code has displayed a good accuracy for simple problems and seems to correctly capture all the basic mechanisms in more complex simulations. The necessity of very refined meshes has been stressed in this work. Adaptive mesh refinement should be even more useful in the future to obtain numerical results in a reasonable computational time. 


\section{References}

Almgren, A., Bell, J., Crutchfield, W., 2000. Approximate projection methods: part I. inviscid analysis. SIAM Journal on Scientific Computing 22, 11391159.

Aulisa, E., Manservisi, S., Scardovelli, R., Zaleski, S., 2007. Interface reconstruction with least-squares fit and split advection in three-dimensional cartesian geometry. J. Comput. Phys. 225, 2301-2319.

Bell, J., Colella, P., Glaz, H., 1989. A second-order projection method for the incompressible Navier-Stokes equations. J. Comput. Phys. 85, 257-283.

Ben Rayana, F., 2007. Contribution à l'étude des instabilités interfaciales liquide-gaz en atomisation assistée et taille de gouttes. Ph.D. thesis, Institut National Polytechnique de Grenoble.

Ben Rayana, F., Cartellier, A., Hopfinger, E., 2006. Assisted atomization of a liquid layer: investigation of the parameters affecting the mean drop size prediction. In: Proc. ICLASS 2006, Aug. 27-Sept.1, Kyoto Japan. Academic Publ. and Printings, iSBN4-9902774-1-4.

Bianchi, G. M., Pelloni, P., Toninel, S., Scardovelli, R., Leboissetier, A., Zaleski, S., 2005. A quasi-direct 3D simulation of the atomization of high-speed liquid jets. In: Proceedings of ICES05, 2005 ASME ICE Division Spring Technical Conference. Chicago, Illinois, USA, April 5-7, 2005.

Boeck, T., Li, J., López-Pagés, E., Yecko, P., Zaleski, S., 2007. Ligament formation in sheared liquid-gas layers. Theor. Comput. Fluid Dyn. 21, 59-76.

Boeck, T., Zaleski, S., 2005. Viscous versus inviscid instability of two-phase mixing layers with continuous velocity profile. Phys. Fluids 17, 032106.

Brackbill, J., Kothe, D. B., Zemach, C., 1992. A continuum method for modeling surface tension. J. Comput. Phys. 100, 335-354.

Chorin, A., 1969. On the convergence of discrete approximations to the NavierStokes equations. Mathematics of Computation 23 (106), 341-353.

Cummins, S., Francois, M., Kothe, D., 2005. Estimating curvature from volume fractions. Computers and Structures 83, 425-434.

De Villiers, E., Gosman, A. D., Weller, H., 2004. Large eddy simulation of primary diesel spray atomisation. SAE Journal of Engines 113, 193-206.

DeBar, R., 1974. Fundamentals of the KRAKEN code. Tech. rep., California Univ., Livermore (USA). Lawrence Livermore Lab.

Dombrowski, N., Johns, W. R., 1963. The aerodynamic instability and disintegration of viscous liquid sheets. Chemical Engineering Science 18, 203-214. 
Faeth, G., Hsiang, L.-P., Wu, P.-K., 1995. Structure and breakup properties of sprays. Int. J. Multiphase Flow 21, 99-127, supplement issue.

Francois, M. M., Cummins, S. J., Dendy, E. D., Kothe, D. B., Sicilian, J. M., Williams, M. W., 2006. A balanced-force algorithm for continuous and sharp interfacial surface tension models within a volume tracking framework. J. Comput. Phys. 213, 141-173.

Gorokhovski, M., Herrmann, M., 2008. Modeling Primary Atomization. Annu. Rev. of Fluid Mech., 343-366.

Greaves, D., 2004. A quadtree adaptive method for simulating fluid flows with moving interfaces. J. of Comp. Phys. 194, 35-56.

Greaves, D., 2006. Simulation of viscous water column collapse using adapting hierarchical grids. Inter. J. for Numer. Meth. in Fluids 50, 693-711.

Gueyffier, D., Nadim, A., Li, J., Scardovelli, R., Zaleski, S., 1998. Volume of fluid interface tracking with smoothed surface stress methods for three-dimensional flows. J. Comput. Phys. 152, 423-456.

Harvie, D., Davidson, M., Rudman, M., 2006. An analysis of parasitic current generation in volume of fluid simulations. Applied Mathematical Modelling 30, 1056-1066.

Ho, C. M., Huerre, P., 1984. Perturbed free shear layers. Annu. Rev. of Fluid Mech. 16, 365-424.

Jeandel, X., Dumouchel, C., 1999. Influence of the viscosity on the linear stability of an annular liquid sheet. Inter. J. of Heat Fluid Flow 20, 499- 506.

Jeong, J., Yang, D., 1998. Finite element analysis of transient fluid flow with free surface using VOF (volume-of-fluid) method and adaptive grid. Int. J. for Numer. Meth. in Fluids 26, 1127-1154.

Keller, F. X., Li, J., Vallet, A., Vandromme, D., Zaleski, S., 1994. Direct numerical simulation of interface breakup and atomization. In: Yule, A. J. (Ed.), Proceedings of ICLASS94. Begell House, New York, pp. 56-62.

Kohno, H., Tanahashi, T., 2004. Numerical analysis of moving interfaces using a level set method coupled with adaptive mesh refinement 45, 921-944.

Lafaurie, B., Nardone, C., Scardovelli, R., Zaleski, S., Zanetti, G., 1994. Modelling merging and fragmentation in multiphase flows with SURFER. J. Comput. Phys. 113, 134-147.

Le Moyne, L., Guibert, P., Roy, R., Jeanne, B., 2007. Fluorescent-piv spray/air interaction analysis of high-pressure gasoline injector. In: Proceedings of the International conference on fuel and lubricants Kyoto. SAE - IMechE. 
Li, J., 1995. Calcul d'interface affine par morceaux (piecewise linear interface calculation). C. R. Acad. Sci. Paris, série IIb, (Paris) 320, 391-396.

Malik, M., Sheung-Chi Fan, E., Bussmann, M., 2007. Adaptive VOF with curvature-based refinement. Inter. J. for Num. Meth. in Fluids 55 (7), 693712 .

Marmottant, P., Villermaux, E., 2004. On spray formation. J of Fluid Mech 498, 73-111.

Ménard, T., Beau, P. A., Tanguy, S., Berlemont, A., 2005. Primary breakup: DNS of liquid jet to improve atomization modelling. Comput Meth in Multiphase Flow III, 343-352.

Ménard, T., Tanguy, S., Berlemont, A., 2007. Coupling level set/VOF/ghost fluid methods: Validation and application to 3D simulation of the primary break-up of a liquid jet. Int Jour of Multiphase Flow 33, 510-524.

Min, C., Gibou, F., 2007. A second order accurate level set method on nongraded adaptive Cartesian grids. J of Comput Phys 225 (1), 300-321.

Nikolopoulos, N., Theodorakakos, A., Bergeles, G., 2007. Three-dimensional numerical investigation of a droplet impinging normally onto a wall film. J. Comput. Phys. 225, 322-341.

Noh, W., Woodward, P., 1976. SLIC (simple line interface calculation). In: van de Vooren, A., Zandbergen, P. (Eds.), Proceedings, Fifth International Conference on Fluid Dynamics. Vol. 59 of Lecture Notes in Physics. Springer, Berlin, pp. 330-340.

Oertel, H. J., 1990. Wakes behind bluff bodies. Ann. Rev. of Fluid Mech. 22, $539-564$.

Popinet, S., 2003. Gerris: a tree-based adaptive solver for the incompressible Euler equations in complex geometries. J. Comput. Phys. 190, 572-600.

Popinet, S., 2007. An accurate adaptive solver for surface-tension-driven interfacial flows. J. Comp. Phys. (submitted).

Popinet, S., 2008. http://gfs.sourceforge.net/wiki/index.php.

Popinet, S., Zaleski, S., 1999. A front tracking algorithm for the accurate representation of surface tension. Int. J. Numer. Meth. Fluids 30, 775-793.

Rangel, R., Sirignano, W., 1988. Nonlinear growth of Kelvin-Helmholtz instability: effect of surface tension and density ratio. Phys. Fluids 31, 1845-1855.

Renardy, Y., Renardy, M., 2002. PROST - A parabolic reconstruction of surface tension for the volume-of-fluid method. J. Comput. Phys. 183, 400-421. 
Rider, W. J., Kothe, D. B., 1998. Reconstructing volume tracking. J. Comput. Phys. 141, 112-152.

Roshko, A., 1954. On the development of turbulent wakes from vortex sheets. NACA report 1991.

Scardovelli, R., Zaleski, S., 1999. Direct numerical simulation of free-surface and interfacial flow. Annu. Rev. Fluid Mech. 31, 567-603.

Scardovelli, R., Zaleski, S., 2001. Analytical relations connection linear interfaces and volume fractions in rectangular grids. J. Comput. Phys. 164, 228237.

Sekar, J., May 2005. The effect of liquid viscosity on the instability of a swirling annular liquid sheet. MS Thesis, University of Cincinnati.

Senecal, P. K., Schmidt, D. P., Nouar, I., Rutland, C., Reitz, R., Corradini, M., 1999. Modeling high-speed viscous liquid sheet atomization. Int. J. Multiphase Flow 25, 1073-1097.

Sochnikov, V., Efrima, S., 2003. Level set calculations of the evolution of boundaries on a dynamically adaptive grid. Inter Jour for Numer Meth in Engineering 56, 1913-1929.

Tauber, W., 2002. Numerical Simulations of Atomization Through the Nonlinear Behavior in a Sheared Immiscible Fluid Interface. Ph.D. thesis, University of Michigan.

Tauber, W., Tryggvason, G., 2000. Direct numerical simulation of primary breakup. Comput. Fluid Dynamics J. 9, 594.

Tauber, W., Unverdi, S. O., Tryggvason, G., 2002. The non-linear behavior of a sheared immiscible fluid interface. Phys. Fluids 14, 2871-2885.

Theodorakakos, A., Bergeles, G., 2004. Simulation of sharp gas-liquid interface using VOF method and adaptive grid local refinement around the interface. Intern Jour for Num Meth in Fluids 45, 421-439.

Torrey, M. D., Cloutman, L. D., Mjolsness, R. C., Hirt, C. W., 1985. NASAVOF2D: A computer program for incompressible with free surfaces. Technical Report LA-101612-MS, Los Alamos National Laboratory.

Tröger, R., 2004. Etude des processus d'injection et de formation du mélange diphasique réactif. application à l'injection directe dans les moteurs conventionnels et alternatifs. Ph.D. thesis, Paris Pierre et Marie Curie.

Wang, J., Borthwick, A., Taylor, R., 2004. Finite-volume-type VOF method on dynamically adaptive quadtree grids. International Journal for Numerical Methods in Fluids 45 (5), 485-508. 
Yecko, P., Zaleski, S., 1999. Two-phase shear instability: waves, fingers and drops. Annals of the New York Academy of Sciences 898, 127-143.

Yecko, P., Zaleski, S., 2005. Transient growth in two-phase mixing layers. J. Fluid Mech. 528, 43-52.

Yecko, P., Zaleski, S., Fullana, J.-M., 2002. Viscous modes in two-phase mixing layers. Phys.Fluids 14, 4115-4122.

Zaleski, S., Li, J., Scardovelli, R., Zanetti, G., 1997. Direct simulation of multiphase flows with density variations. In: IUTAM colloquium "Variable Density Low Speed Turbulent Flows", Marseilles, 8-10 July 1996. Kluwer, pp. 51-58.

Zheng, X., Lowengrub, J., Anderson, A., Cristini, V., 2005. Adaptive unstructured volume remeshing-II: Application to two-and three-dimensional levelset simulations of multiphase flow. Jour of Comput Phys 208 (2), 626-650. 